Title: Synchronization and interaction of proline, ascorbate and oxidative stress pathways under abiotic stress combination in tomato plants

Authors: María Lopez-Delacalle ${ }^{1}$, Christian J Silva ${ }^{2}$, Teresa C Mestre ${ }^{1}$, Vicente Martinez $^{1}$, Barbara Blanco-Ulate ${ }^{2^{*}}$, Rosa M Rivero ${ }^{1 *}$

(*) Corresponding authors

\title{
Addresses of the institutions:
}

${ }^{1}$ CEBAS-CSIC, Campus Universitario de Espinardo, 30100, Murcia, Spain.

2 Department of Plant Sciences, University of California Davis, Davis, CA, 95616, USA.

\section{Author's emails:}

María Lopez-Delacalle: mlopez@ cebas.csic.es

Christian J Silva: cjsilva@ ucdavis.edu

Teresa C Mestre: tmestre@ cebas.csic.es

Vicente Martinez: vicente@ cebas.csic.es

Barbara Blanco-Ulate: bblanco@ucdavis.edu

Rosa M Rivero: rmrivero@ cebas.csic.es

Date of Submission: June 30th 2020

$\mathbf{N}^{0}$ Figures: 6

Word Count:

Supplemental material: 7 supplemental tables

3 supplemental figures.

\section{HIGHLIGHTS:}

- The combination of salinity and heat causes a unique reprogramming of tomato metabolic pathways by changing the expression of specific genes and metabolic features.

- Proline and ascorbate pathways act synchronously to maintain cellular redox homeostasis

- Key transcription factors from the basic Leucine Zipper Domain (bZIP), Zinc Finger Cysteine-2/Histidine-2 (C2H2) and Trihelix families were identified as putative regulators of the identified up-regulated genes under salinity and heat combination. 


\begin{abstract}
Adverse environmental conditions have a devastating impact on plant productivity. In nature, multiple abiotic stresses occur simultaneously, and plants have evolved unique responses to cope against this combination of stresses. Here, we coupled genome-wide transcriptional profiling and untargeted metabolomics with physiological and biochemical analyses to characterize the effect of salinity and heat applied in combination on the metabolism of tomato plants. Our results demonstrate that this combination of stresses causes a unique reprogramming of metabolic pathways, including changes in the expression of 1,388 genes and the accumulation of 568 molecular features. Pathway enrichment analysis of transcript and metabolite data indicated that the proline and ascorbate pathways act synchronously to maintain cellular redox homeostasis, which was supported by measurements of enzymatic activity and oxidative stress markers. We also identified key transcription factors from the basic Leucine Zipper Domain (bZIP), Zinc Finger Cysteine-2/Histidine-2 (C2H2) and Trihelix families that are likely regulators of the identified up-regulated genes under salinity+heat combination. Our results expand the current understanding of how plants acclimate to environmental stresses in combination and unveils the synergy between key cellular metabolic pathways for effective ROS detoxification. Our study opens the door to elucidating the different signaling mechanisms for stress tolerance.
\end{abstract}

Keywords: salinity, heat, protein oxidation, lipid peroxidation, reactive oxygen species, plant stress. 


\section{INTRODUCTION}

2 Multiple environmental factors such as salinity, high temperatures, cold, or drought

3 cause abiotic stresses in plants, which result in large agricultural losses worldwide,

4 estimated to be around \$14-19 million (Rivera et al., 2017). Under field conditions,

5 different abiotic stressors usually occur at the same time; for example, it is common that

6 high temperatures coexist with highly saline soils or water scarcity. Studies in the last

7 decade have shown that plant responses to combined abiotic stresses are unique and

8 cannot be deduced from the study of plants subjected to each stress separately (Mittler,

9 2006; Miller et al., 2010; Rivero et al., 2014; Anjum et al., 2019; Sehgal et al., 2019;

10 Lopez-Delacalle et al., 2020; Zandalinas et al., 2020).

11 Many metabolic mechanisms act in concert during the plant's response to abiotic stress,

12 including rapid changes in gene expression, ionic adjustment, and activation and

13 inactivation of proteins that carry out the synthesis and degradation of compounds used

14 for cell signaling and protection (e.g., osmoprotectants and antioxidants), among others

15 (Rivero et al., 2014; Zushi et al., 2014; Zandalinas et al., 2020). Proline has been widely

16 reported to act as an osmoprotectant in plant defense against certain stress conditions,

17 such as drought and salinity (Rivero et al., 2004b, 2014)(Rivero et al., 2004b, 2014;

18 Martinez et al., 2018). Under heat stress, plants synthesize proline, as reported by the

19 induction of pyrroline-5-carboxylate synthase (P5CS) and the subsequent accumulation

20 of the amino acid (Rivero et al., 2004b; Torres et al., 2006). Shalata \& Neumann (2001)

21 have also reported that under salinity, proline accumulation can improve plant salt

22 tolerance and reduce oxidative damage by decreasing lipid peroxidation in tomato 23 plants.

24 Stress conditions cause the accumulation of reactive oxygen species (ROS), which are

25 known to induce oxidative stress (e.g., lipid peroxidation) and serve as signaling

26 molecules in plants (Suzuki et al., 2012; Kollist et al., 2019). Plants accumulate

27 antioxidants, such as ascorbate (ASC), glutathione (GSH), carotenoids, tocopherols, and

28 flavonoids, and activate enzymatic reactions to maintain cell homeostasis under

29 increasing oxidative conditions. The ASC/GSH cycle is critical for detoxifying ROS

30 from plant cells. Briefly, the hydrogen peroxide $\left(\mathrm{H}_{2} \mathrm{O}_{2}\right)$ generated by detoxification of

31 superoxide radicals is further converted to $\mathrm{H}_{2} \mathrm{O}$ and $\mathrm{O}_{2}$ by ASC peroxidase (APX), the

32 first enzyme of the ASC/GSH cycle, using ASC as an electron donor (Noctor and Foyer,

33 1998). Because ASC is considered the first antioxidant line of defense in $\mathrm{H}_{2} \mathrm{O}_{2}$ 
34 detoxification (Foyer and Noctor, 2011; Akram et al., 2017), it is expected that plants

35 with a high cellular accumulation of this compound will have a greater tolerance to 36 oxidative stress. In fact, tomato seeds treated with ascorbic acid have been shown to 37 have better tolerance to salinity stress, improved germination, and better growth 38 parameters (Sayed, 2013). We have previously reported (Rivero et al. 2004) that 39 enzymes that belong to the ASC/GSH cycle were inhibited in tomato plants under high 40 temperature, leading to $\mathrm{H}_{2} \mathrm{O}_{2}$ accumulation and inhibition of plant growth and yield. In 41 addition to its importance in ROS detoxification, the cellular content of GSH and ASC 42 improves osmoregulation, efficient use of water, photosynthetic activity, and general 43 parameters of plant productivity (Noctor and Foyer, 1998; Meyer, 2008; Foyer and 44 Noctor, 2011).

45 Plants need to rapidly regulate and fine-tune their responses to stress in order to 46 maximize energy expenditure in adverse conditions. Transcription factors (TFs) are 47 considered key components in the control of abiotic stress signaling (Schmidt et al., 48 2012; Castelán-Muñoz et al., 2019); however, little is known about their role in stress 49 combination. Just recently, a report by Zandalinas et al. (Zandalinas et al., 2020) found 50 that Arabidopsis plants induced a unique set of TFs when subjected to different abiotic 51 stress combinations, and that those genes were relatively unique across stress 52 conditions.

53 Here we hypothesize that the combination of salinity and heat induces a unique 54 physiological response in tomato plants by activating specific regulatory and metabolic 55 pathways that act synergistically to maintain cell redox homeostasis. In this work, we 56 analyze how the combination of salinity and heat affects the transcriptome and 57 metabolome of tomato plants in order to find the unique elements that are differentially 58 regulated under these stress conditions and that may be key in ROS detoxification and, 59 thus, plant tolerance to abiotic stress combination. 
60 MATERIALS AND METHODS

61 Plant material, experimental design, and growth conditions

62 Solanum lycopersicum L cv. Boludo (Monsanto) seeds were germinated in vermiculite 63 under optimal and controlled conditions in a growth chamber (chamber A). These 64 conditions were: a photoperiod of $16 / 8$ hours of day/night with a light intensity of 500 $65 \mu \mathrm{mol} \mathrm{m} \mathrm{s}^{-1}$, a relative humidity (RH) between 60 and $65 \%$ and a temperature of $25^{\circ} \mathrm{C}$. 66 Subsequently, when the plants had at least two true leaves, they were transplanted to an 67 aerated hydroponic system containing a modified Hoagland solution and grown under 68 these conditions for one week. The nutrient solution had the following composition: $69 \mathrm{KNO}_{3}(3 \mathrm{mM}), \mathrm{Ca}\left(\mathrm{NO}_{3}\right)_{2}(2 \mathrm{mM}), \mathrm{MgSO}_{4}(0.5 \mathrm{mM}), \mathrm{KH}_{2} \mathrm{PO}_{4}(0.5 \mathrm{mM})$, Fe-EDTA (10 $70 \mu \mathrm{M}), \mathrm{H}_{3} \mathrm{BO}_{3}(10 \mu \mathrm{M}), \mathrm{MnSO}_{4} \cdot \mathrm{H}_{2} \mathrm{O}(1 \mu \mathrm{M}), \mathrm{ZnSO}_{4} \cdot 7 \mathrm{H}_{2} \mathrm{O}(2 \mu \mathrm{M}), \mathrm{CuSO}_{4} \cdot 5 \mathrm{H}_{2} \mathrm{O}(0.5$ $71 \mu \mathrm{M})$, and $\left(\mathrm{NH}_{4}\right)_{6} \mathrm{Mo}_{7} \mathrm{O}_{24} \cdot 4 \mathrm{H}_{2} \mathrm{O}(0.5 \mu \mathrm{M})$ (Hoagland and Arnon, 1950). The electric conductivity (EC) and $\mathrm{pH}$ of the nutrient solution were measured and maintained within

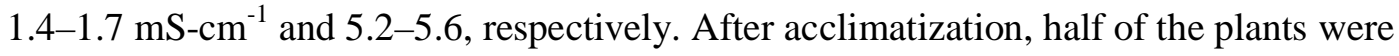
transferred to a twin chamber whose temperature was previously set at $35^{\circ} \mathrm{C}$ (chamber B). In both twin chambers, a saline concentration in the nutrient solution of $75 \mathrm{mM}$ $\mathrm{NaCl}$ was added to half of the plants. Therefore, four different conditions were obtained: control $\left(25^{\circ} \mathrm{C} 0 \mathrm{mM} \mathrm{NaCl}\right)$, salinity $\left(25^{\circ} \mathrm{C}\right.$ and $\left.75 \mathrm{mM} \mathrm{NaCl}\right)$, heat $\left(35^{\circ} \mathrm{C} 0\right.$ $\mathrm{mM} \mathrm{NaCl})$, and salinity and heat $\left(35^{\circ} \mathrm{C}\right.$ and $\left.75 \mathrm{mM} \mathrm{NaCl}\right)$. Plants were kept under these conditions for a period of 14 days. After this time, six plants from each treatment were sampled for subsequent analysis.

\section{Measurements of photosynthetic parameters}

83 Photosynthetic parameters were determined on a fully-expanded, metabolically-mature middle leaf in all plants. These data were taken with a gas exchange system (LI-COR 6400, Li-Cor) at the beginning (day 0), the middle (day 7), and at the end of the experiment (day 14). The conditions established in the LI-COR were: $1000 \mu \mathrm{mol}$ photons $\mathrm{m}^{-2} \mathrm{~s}^{-1}$ and $400 \mu \mathrm{mol} \mathrm{mol}{ }^{-1} \mathrm{CO}_{2}$. The leaf temperature was maintained at $25^{\circ} \mathrm{C}$ for control and salinity treatment plants, and at $35^{\circ} \mathrm{C}$ for plants in the high temperature, and the combination of high temperature and salinity treatments. The leaf-air vapor pressure deficit was maintained between 1-1.3 $\mathrm{kPa}$. With this analysis, the device 
92 end of the experiment, the leaves, stem, and root were separated, and the fresh weight

$93(\mathrm{FW})$ of each part of the plant was determined separately.

95 Quantification of oxidative stress-related markers

$96 \mathrm{H}_{2} \mathrm{O}_{2}$ accumulation

$97 \mathrm{H}_{2} \mathrm{O}_{2}$ was extracted as described by Yang et al. (2007), with some modifications, which

98 are fully described in García-Martí et al. (García-Martí et al., 2019). The samples were

99 used for the future determination of $\mathrm{H}_{2} \mathrm{O}_{2}$ concentration and lipid peroxidation. $\mathrm{H}_{2} \mathrm{O}_{2}$

100 was quantified as described by MacNevin and Urone (1953).

101 Lipid peroxidation

102 For lipid peroxidation determination, malondialdehyde (MDA) was used, which is a 103 product of the peroxidation of membrane lipids. The same enzyme extract as the one 104 utilized for the determination of $\mathrm{H}_{2} \mathrm{O}_{2}$ was used. The procedure was described by $\mathrm{Fu}$ 105 and Huang (2001).

106 Antioxidant capacity

107 Regarding antioxidant capacity, it was carried out according to the protocol by Koleva 108 et al. (2002). The remaining amount of 2,2-diphenyl-1-picrylhydrazyl (DPPH), 109 measured at a certain time, is inversely proportional to the antioxidant capacity of the 110 substances present in the sample. Results are expressed as \% Radical Scavenging

111 Activity (RSA), or percentage of free radical scavenging activity.

112 Protein oxidation

113 Protein oxidation was assayed as according to Reznick and Packer (1994). PCO groups 114 react with 2,4-dinitrophenylhydrazine (DNPH) to generate chromophoric 115 dinitrophenylhydrazones, which can be recorded with a spectrophotometer. The 116 absorbance was measured at $360 \mathrm{~nm}$, using the molar extinction coefficient of DNPH $1172.2 \times 10^{4} \mathrm{M}^{-1} \mathrm{~cm}^{-1}$.

\section{RNA extraction and sequencing}

119 Total RNA was extracted from $1 \mathrm{~g}$ of frozen tomato leaves using TRI-Reagent (Sigma-

120 Aldrich) and following the manufacturer's instruction. The quantity and quality of RNA 121 were determined using a NanoDrop 3300 fluorospectrometer (Thermo Scientific 122 Instruments, USA). Three biological replications for each treatment were used for total 123 RNA extraction and sequencing. For each RNA sample, mRNA was enriched using a 124 Dynabeads mRNA purification kit (Invitrogen), then the samples were sent to BGI- 
125 Shenzhen (hereafter 'BGI', China) for RNA sequencing. Sequencing was carried out on

126 a HiSeq2000 according to the Illumina protocols for $90 \square \times \square 2$ pair-end sequencing

127 covering a read length of $100 \mathrm{bp}$. An average of $10 \mathrm{~Gb}$ clean data per sample was

128 generated after filtering to ensure a complete set of expressed transcripts with sufficient

129 coverage and depth for each sample.

130

131 Bioinformatics pipeline

132 RNA sequencing and data processing

133 Raw sequencing reads were trimmed for quality and adapter sequences using 134 Trimmomatic v0.33 (Bolger et al., 2014) were used with the following parameters: 135 maximum seed mismatches $=2$, palindrome clip threshold $=30$, simple clip threshold $=$ 13610 , minimum leading quality $=3$, minimum trailing quality $=3$, window size $=4$, 137 required quality $=15$, and minimum length $=36$. Trimmed reads were mapped using 138 Bowtie2 (Langmead and Salzberg, 2012) to the tomato transcriptome (SL4.0 release; 139 http://solgenomics.net). Count matrices were made from the Bowtie2 results using 140 sam2counts.py v0.91 (https://github.com/vsbuffalo/sam2counts/). A summary of the 141 quality assessment and mapping results can be found in Supplemental Table S1. The 142 raw sequencing reads and the read mapping count matrices are available in the National 143 Center for Biotechnology Information Gene Expression Omnibus database under the 144 accession GSE152620.

\section{Differential expression analysis}

146 The Bioconductor package DESeq2 (Love et al., 2014) was used to perform 147 normalization of read counts and differential expression analyses for various treatment 148 comparisons. Differentially expressed (DE) genes for each comparison were those with 149 an adjusted p-value of less than or equal to 0.05 .

\section{Functional annotation and enrichment analyses}

151 Basic functional annotations for genes were determined with the Automated 152 Assignment of Human Readable Descriptions (AHRD) provided in the SL4.0 build of 153 the tomato genome. KEGG annotations were determined using the KEGG Automatic 154 Annotation Server (Moriya et al., 2007). Enrichments were conducted via Fisher's exact 155 test with p-values adjusted using the Benjamini and Hochberg method (Benjamini and 156 Hochberg, 1995).

157 Promoter motif analysis 
158 Binding motifs for tomato transcription factors were obtained from the Plant 159 Transcription Factor Database (http://planttfdb.cbi.pku.edu.cn/). Promoter sequences 160 defined as 1000 base pairs upstream from the transcription start site of each gene were 161 obtained using the 'flank' function in bedtools v2.29.2 162 (https://bedtools.readthedocs.io/en/latest/). Enrichment of transcription factor binding 163 motifs on the promoter sequences of up-regulated genes was performed using the 164 Analysis of Motif Enrichment tool in MEME-Suite (McLeay and Bailey, 2010) using 165 all non-up-regulated tomato genes as the control sequences, the average odds score 166 scoring method and Fisher's exact test.

\section{Metabolomics analysis}

169 Six biological replications of frozen tomato leaves per treatment were used for the 170 metabolomics analysis. One gram of this frozen plant material was extracted in 171 methanol: water (3:1 v/v) as described previously in Martinez et al. (2016). Agilent 172 MassHunter Qualitative analysis software v 6.00 (Agilent Technologies, Palo Alto, 173 USA) was used to obtain an initial peak processing (Supplemental Fig. S1). Then, 174 XCMS online software (www.xcmsonline.scripts.edu) which incorporates CAMERA (a 175 Bioconductor package to extract spectra, annotate isotopes and adduct peaks, among 176 other functions) in its analysis, was implemented in our curated raw data 177 (Supplemental Table S2). A second level of statistical analysis was carried out, 178 consisting of data normalization of the peaks obtained for each treatment against the 179 control, and a t-test followed by an ANOVA analysis. Then, $\log _{2}$ of the fold-change was 180 calculated and all the molecular features with a $\mathrm{P}_{a d j}$ adjusted greater than 0.05 and a $\log _{2}$ 181 fold change (FC) greater than -1 or smaller than 1 were eliminated from the analyses 182 (Supplemental Table S3). All the molecular features that remained after these 183 restricted statistical analyses were compared among the different treatments applied 184 (supplemental Table S3; Euler diagram Fig 3B).

185 The metabolite identification of the molecular features of interest for this study was 186 performed using a mathematical search based on the predicted elemental composition 187 through some of the most important open-source databases (MOTO, KNApSAcK, 188 KOMOCS, MassBank, ARMeC and METLIN) within a mass tolerance of $10 \mathrm{ppm}$. 189 Then, the isotope ratio (IR) and retention time (rt) from the different metabolites 190 identified unequivocally were checked again across the different databases mentioned 
191 above. Identified metabolites that remained after this filtering were labeled accordingly

192 and highlighted in yellow in Supplemental Table S4. The concentration of the

193 compounds that showed significant differences $\left(\mathrm{P}_{a d j}<0.05\right.$ and $\left.\log _{2} \mathrm{FC}>2\right)$ under

194 salinity and heat combination as compared to control plants and which were of interest

195 in this study were plotted in a box-and-whisker type plot using XCMS online

196 (Supplemental Figs. S2 and S3).

197

198 Enzymatic activities

199 Crude extract

200 All enzymatic activities were extracted according to the procedure described by Torres

201 et al. (2006).

202 Superoxide dismutase (SOD)

203 SOD activity was assayed as described previously by McCord JM (1969). SOD activity

204 was expressed as units of SOD $\left(\mathrm{mg} \mathrm{prot}^{-1}(\mathrm{~min})^{-1}\right.$, a unit which indicates the amount of

205 enzyme needed to neutralize one unit of xanthine oxidase.

206 Catalase (CAT)

207 CAT activity was calculated using the extinction coefficient of $39.4 \mathrm{mM}^{-1} \mathrm{~cm}^{-1}$ as

208 described by Aebi (1984). CAT activity was expressed as $\mu \mathrm{mol}$ of reduced $\mathrm{H}_{2} \mathrm{O}_{2}$ (mg $209 \operatorname{prot}^{-1}(\mathrm{~min})^{-1}$.

210 Ascorbate peroxidase (APX) Dehidroascorbate reductase (DHAR) and

211 Monodehidroascorbate reductase (MDHAR)

212 APX, DHAR and MDHAR activities were assayed as described by Miyake and Asada

213 (1992). The rate of reaction was calculated using a molar extinction coefficient of 2.8

$214 \mathrm{mM}^{-1} \mathrm{~cm}^{-1}$. APX activity was expressed as $\mu \mathrm{mol}$ of reduced ascorbic acid (mg prot) ${ }^{-1}$

$215(\min )^{-1}$.

216 Glutathione peroxidase (GPX)

217 GPX activity was carried out using the Glutathione Peroxidase Assay Kit (Abcam, Ref.

218 ab102530, Cambridge, UK) considering the decrease of NADPH at $340 \mathrm{~nm}$, using an

219 extinction coefficient of $6.22 \mathrm{mM}^{-1} \mathrm{~cm}^{-1}$.

220 Glutathione reductase (GR)

221 GR activity was assayed through the non-enzymatic NADPH oxidation (Halliwell and

222 Foyer, 1976). The activity was determined by measuring the decrease in the reaction

223 rate at $340 \mathrm{~nm}$ and was calculated from the $6.22 \mathrm{mM}^{-1}$ extinction coefficient. 


\section{Protein concentration in the enzyme extract}

Proteins were quantified with the Bradford method (Bradford, 1976), in which a volume of Bradford Reagent reagent (BioRAd, Catalog No. 30214) was added to an aliquot of the enzyme extract. The absolute values as well as the calculated $\log _{2}$ of the data normalized against control plants of all the enzymatic activities assayed can be found in

\section{Supplementary Table S5.}

\section{Statistical analysis}

Statistical analysis for $\mathrm{FW}$, photosynthetic parameters, $\mathrm{H}_{2} \mathrm{O}_{2}$ concentration, MDA content, protein oxidation, and enzymatic activities were performed with an analysis of variance with $\mathrm{p}$-value $<0.05$ set as the cut-off value, as indicative of significant differences, followed by a Duncan test and a t-test when necessary. Transcriptomics and metabolomics statistical analysis was performed as described above.

\section{RESULTS}

Tomato plants grown under the combination of salinity showed a better performance in the photosynthetic parameters as compared to salinity alone

Eighty-four tomato plants were grown in two independent chambers using four different environmental conditions: $25{ }^{\circ} \mathrm{C}$ and $0 \mathrm{mM} \mathrm{NaCl}$ (control), $25{ }^{\circ} \mathrm{C}$ and $75 \mathrm{mM} \mathrm{NaCl}$ (salinity), $35{ }^{\circ} \mathrm{C}$ and $0 \mathrm{mM} \mathrm{NaCl}$ (heat), and $35{ }^{\circ} \mathrm{C}$ and $75 \mathrm{mM} \mathrm{NaCl}$ (salinity + heat) for 14 days (Fig. 1A). Fresh weight was recorded at the end of the experiment (Fig. 1B). Salinity and the salinity + heat resulted in a significant reduction of biomass when compared to control plants, whereas the heat-treated plants did not differ significantly from the controls. Interestingly, when salinity and heat were applied simultaneously, the growth was significantly improved with respect to salinity up to about $18 \%$. Photosynthetic parameters were also measured at 0 days, and after 7 and 14 days after the start of the treatments, as stress physiological markers (Figs. 1C-F). In our experiments, $\mathrm{CO}_{2}$ assimilation rate was highly inhibited after 7 days under salinity as compared to control plants, with an inhibition of 50\% at 7 days, and $70 \%$ at 14 days with respect to control plants (Fig. 1C). The other stress treatments applied (heat and salinity + heat) did not differ significantly with respect to the values obtained in control plants during the entire experiment, contrary to that observed under salinity. Control plants had a transpiration rate and a stomatal conductance that were practically constant 
257 during the entire experiment, whereas plants subjected to heat and salinity + heat

258 treatments showed a significant increase in transpiration rate (41\%) and stomatal

259 conductance (29\%) at 7 days, which was maintained until the end of the experiment

260 (Figs. 1D and 1E). Contrarily, the salinity treatment led to a significant reduction in the 261 transpiration rate and the stomatal conductance at day 7 from the start of the treatment 262 until the end. In this regard, the salinity + heat treatment showed a significant 263 improvement in the photosynthetic parameters as compared to salinity alone. Curiously, 264 no differences were found between salinity, heat, and the combination of both stresses

265 for water use efficiency (WUE, Fig. 1F), but all the treatments showed a significant 266 decrease in this parameter as compared to control plants.

Salinity and heat combination induced a specific transcriptional response and pathway activation

An RNAseq study was performed to identify specific biochemical pathways or molecular functions that could explain the different physiological responses of the tomato plants to the salinity, heat, and salinity + heat treatments. RNA was sequenced from three biological replicates from each treatment, including control plants. A principal component analysis of the normalized reads revealed that all samples clustered according to treatment, which validated the unique transcriptional reprograming caused by each stress condition (Fig. 2A). Then, a differential expression analysis was performed to determine the individual genes affected by each treatment when compared to the control. A total of 15,852 genes were found to be differentially expressed $\left(P_{a d j}<\right.$ 0.05) across all three treatments (Supplemental Table S6). A comparison of both upand down-regulated genes from each of the three treatments further confirmed that each treatment resulted in a high number of unique differentially expressed genes (Fig. 2B). Most notably, it was found that 1,388 (7.32\% of the total) were differentially expressed only for salinity + heat, with 923 genes up-regulated and 465 genes down-regulated by this stress combination (Fig. 2B).

285 To identify important functions activated in response to each stress, an enrichment 286 analysis of the significantly up-regulated genes was conducted using pathway 287 annotations from the Kyoto Encyclopedia of Genes and Genomes (KEGG; 288 Supplemental Table S7). A total of 27 pathways were found to be enriched $\left(P_{a d j}<\right.$ 
290 the genes themselves, enriched pathways were largely unique, with all but three

291 pathways (glutathione metabolism (sly00480), protein processing in the ER (sly04141),

292 and spliceosome (sly03040)) being enriched in just one of the three treatments. Most

293 interestingly, the salinity + heat treatment resulted in the upregulation of genes

294 belonging to two main metabolic pathways, ASC and aldarate metabolism (sly00053)

295 and arginine and proline metabolism (sly00330), which were not enriched in either of

296 the individual stress treatments, suggesting that the combination of stresses induced

297 specific changes in plant metabolism that in turn led to variation in the physiological

298 responses of the plants.

299 The salinity and heat combination showed a unique metabolic profile with the 300 enrichment of specific pathways

301 A metabolomics study was carried out to identify molecular features that were common

302 or unique to the simple or combined stresses and to validate the RNAseq results. Our

303 main interest mainly resided in those that were specifically accumulated under the

304 combination of salinity and heat. A total of 3,338 molecular features showed significant

$305\left(P_{a d j}<0.05\right.$ and a $\log _{2}<-1$ or $\left.\log _{2}>1\right)$ changes across the three stress conditions.

306 Similar to the RNAseq analyses, each stress condition showed a unique metabolic

307 profile (Fig. 3A; Supplemental Tables S3 and S4). Only 208 molecular features were

308 commonly altered by all the treatments, which represented $6.30 \%$ of the total. When the

309 combination of salinity and heat was applied, a total of 568 molecular features $(17.19 \%$

310 of the total) were significantly and specifically accumulated with respect to the control

311 (Fig. 3B). Salinity + heat caused reprogramming of multiple metabolic pathways,

312 observed as a similar number of molecular features that were up- or down-regulated,

313337 and 208, respectively, when compared to the control (Fig. 3C). Pathway enrichment

314 analysis of the up-regulated molecular features revealed that four biochemical pathways

315 (i.e., ASC and aldarate metabolism, purine metabolism, arginine and proline

316 metabolism and arginine biosynthesis) were significantly altered in tomato under the

317 combination of salinity and heat (Fig. 3D and Supplementary Table S4,

318 Supplementary Figs. S2 and S3). In agreement with the RNAseq data, the ASC and

319 aldarate metabolism and the arginine and proline metabolism were among the most

320 significantly enriched pathways. 
322 The integration of transcriptomics and metabolomics revealed that the proline and

\section{ASC pathways are interconnected for ROS homeostasis}

324 The RNAseq and metabolomics data was combined with measurements of enzymatic

325 activity to obtain a detailed picture of the changes in the ASC and aldarate, and arginine

326 and proline metabolic pathways caused by the combination of salinity and heat stresses

327 (Fig. 4). The first observation was that proline appears to be degraded in favor of 4-

328 hydroxyproline and L-glutamate-5-semialdehyde accumulation, with the concomitant

329 up-regulation of prolyl 4-hydroxylase (P4HA) and pyrroline-5-carboxylate reductase

330 (PROC), as well as the down-regulation of proline dehydrogenase (PRODH). The

331 accumulation of L-glutamate-5-semialdehyde was also likely derived from ornithine

332 through the up-regulation of arginase (ARG) and ornithine aminotransferase (ROCD).

333 In summary, proline was not differentially accumulated under the combination of

334 salinity + heat as compared to controls. Instead, 4-hydroxyproline and L-glutamate-5-

335 semialdehyde, two proline-derivative compounds, significantly accumulated in tomato

336 leaves under stress combination.

337 ASC significantly accumulated under combined salinity and heat stress in tomato. Its

338 synthesis from UDP-glucose or myo-inositol results in the precursor D-glucuronate,

339 which also increased under salinity + heat, in part due to the down-regulation of

340 glucuronokinase transcript (GLCAK) through glucoronate-1P synthesis and to the up-

341 regulation of one copy of the inositol oxygenase (MIOX). The levels of L-gulose and L-

342 gulonate also increased under the combination of stresses, which seemed to favor ASC

343 accumulation. The high ASC levels observed in tomato plants under stress combination

344 could also be due to the degradation of the GDP-L-galactose and L-galactose-1P, since

345 GDP-L-galactose phosphorylase (VTC2-5) and L-galactose 1-phosphate phosphatase

346 (VTC4) were up-regulated under these conditions (Fig. 4). ASC is known to detoxify

347 ROS through the Halliwell-Asada cycle. Remarkably, this pathway was highly

348 represented among the differentially expressed genes and the significant molecular

349 features altered by salinity + heat (Figs. 2-3). These results were also supported by the

350 enzymatic activities of the proteins encoded by those transcripts (Fig. 4). Superoxide

351 dismutases (SOD1 and SOD2), involved in cell ROS detoxification, were up-regulated

352 at the transcript and activity levels, leading to the conversion of $\mathrm{O}_{2}{ }^{-*}$ to $\mathrm{H}_{2} \mathrm{O}_{2}$. Then,

$353 \mathrm{H}_{2} \mathrm{O}_{2}$ can be detoxified by catalase (CAT) or by ASC peroxidase (APX) through the

354 ASC/GSH pathway. CAT was not differentially expressed in the RNAseq analysis and 
355 the enzyme activity was inhibited by stress combination. Several APX homologs were

356 up-regulated at the transcript level and, more importantly, its enzymatic activity was

357 very high $\left(\log _{2}=1.97\right.$, Supplemental Table S5) under salinity + heat. The APX

358 activity generates monodehydroascorbate, which accumulated significantly in our

359 experiments. Monodehydroascorbate spontaneously forms dehydroascorbate, which is

360 reduced to ASC, once again through the action of dehydroascorbate reductase (DHAR),

361 using glutathione (GSH) as a reducing agent. Tomato plants showed a significant

362 accumulation of glutathione and monodehydroascorbate under combined salinity and

363 heat stress, with a non-significant dehydroascorbate accumulation or DHAR activity.

364 However, the MDAR enzyme, responsible for the regeneration of ASC, was up-

365 regulated at the transcript and enzymatic levels. Lastly, the glutathione peroxidases

366 GPX and PhGPX, responsible for the recovery of lipid peroxidation, were also up-

367 regulated under stress combination. Our results are indicative of a connection between

368 ASC synthesis and oxidative stress-proline metabolism, with the intersection between

369 these pathways found at the 1-pyrroline-5-carboxylate level (Fig. 4).

Plants subjected to salinity and heat combination showed lower oxidative damage than those under salinity alone

373 The proposed coordination between the proline, ASC, and redox pathways may improve

374 the ability of the tomato plants to deal with ROS detoxification. Markers of oxidative

375 stress were evaluated to determine if tomato plants subjected to stress combination

376 displayed a more efficient antioxidant system than those plants grown under individual

377 stresses (Fig. 5). Tomato plants under salinity had the highest levels of $\mathrm{H}_{2} \mathrm{O}_{2}$, with a

378 significant 4-fold increase compared to control plants. However, when salinity and heat

379 were applied in combination, it was found that the $\mathrm{H}_{2} \mathrm{O}_{2}$ content was about $50 \%$ lower

380 than in the salinity treatment (Fig. 5A). A similar trend was observed for lipid

381 peroxidation, an indicator of oxidative damage to cell membranes, with a maximum

382 value found for salinity stress, and an intermediate value found for the salinity + heat

383 stress combination (Fig. 5B). Thus, the stress combination appears to reduce oxidative

384 damage with respect to the salinity treatment. In neither case, the differences between

385 heat stress and control were statistically significant. Interestingly, the antioxidant

386 capacity (Fig. 5C) obtained for plants subjected to salinity was the lowest among all

387 treatments, with a reduction of up to $90 \%$ as compared to the controls. When salinity 
and heat were combined, the antioxidant capacity index was significantly lower than the control but 6-fold higher than salinity. Again, the heat treatment did not show significant differences with respect to the control. Protein oxidation values obtained for the four stress conditions were directly related to $\mathrm{H}_{2} \mathrm{O}_{2}$ and lipid peroxidation, with a positive and significant correlation $\left(\mathrm{H}_{2} \mathrm{O}_{2}\right.$-protein oxidation: $\mathrm{r}=0.992, P_{a d j}<0.001$; lipid peroxidation-protein oxidation: $\left.\mathrm{r}=0.996, P_{a d j}<0.001\right)$. In short, our results indicated that ROS levels were lower when salinity and heat were applied jointly as compared to the salinity treatment alone, which was directly observed as a lower damage to the membrane lipids and to the cellular proteins under abiotic stress combination.

\section{The combined salinity and heat responses are associated with the upregulation of} unique transcription factors families

The high specificity of the tomato plant responses to salinity + heat suggests that a tight regulatory control must be in place to rapidly and efficiently cope with the oxidative damage caused by these conditions. TFs are known to be key players in modulating the expression of genes involved in abiotic stress responses. TFs that may regulate the transcriptional responses to salinity, heat, and/or salinity + heat were identified by evaluating the promoter regions (1000 bp upstream from the transcription start site) of up-regulated genes from each stress condition for overrepresented cis-element motifs (Fig. 6A). Binding sites from a total of 46 TFs belonging to multiple gene families were found to be enriched $\left(P_{a d j}<0.05\right)$ across all treatments. Of these, only 9 TFs were associated with all stress conditions (salinity, heat, and salinity + heat), including three Homeobox-Homeodomain-Leucine Zipper Protein (HB-HD-ZIP) TFs identified. The salinity + heat treatment exhibited five unique TFs, including three from the stressrelated Zinc Finger Cysteine-2/Histidine-2 (C2H2) family.

In contrast to the diverse enrichment results, most enriched TFs did not exhibit significant up-regulation themselves under their associated stress condition. For salinity + heat, only three TFs had significant $\left(P_{a d j}<0.05\right)$ expression levels when compared to the controls (Fig. 6B). These TFs, one each from the basic Leucine Zipper Domain (bZIP), C2H2, and HB-HD-ZIP families, were also all differentially expressed in the salinity treatment, although the bZIP TF (Solyc04g078840) was down-regulated under this treatment, and up-regulated exclusively for salinity + heat. None of these three were 
421 differentially expressed under the heat stress alone. The sequences of the differentially

422 expressed genes from the proline, ASC, and redox pathways (Fig. 4) were evaluated to

423 determine which of the overrepresented cis-element motifs associated with salinity +

424 heat were present in their promoters (Fig. 6A). Most of these genes include binding sites

425 for TFs from the Apetala 2 (AP2), Dof zinc finger protein (C2C2-Dof), and Cysteine-

426 rich Polycomb-like Protein (CPP) families, among others. Binding sites for the single

427 CPP TF, which were highly enriched across all three stress conditions, matched to the

428 promoters of genes from the proline, ASC, and oxidative metabolism pathways,

429 including all four up-regulated copies of $A P X$ genes. Remarkably, binding sites for the

430 single-enriched Trihelix TF, Solyc11g012720, were found only in the promoters of

431 proline metabolism genes. Ultimately, these results suggest that specific sets of TFs

432 coordinate the modulation of proline, ASC, and redox metabolism under salinity + heat

433 stress, which should be further studied to validate their direct or indirect regulatory

434 roles.

\section{DISCUSSION}

437 In the present study, we demonstrated that the combination of heat stress with moderate 438 salinity in tomato plants induced a specific physiological, biochemical, and molecular 439 response that could not be deduced from a single stress application. From the 440 physiological stand point, tomato plants under the combination of salinity and heat grew 441 better than when salinity was applied as a sole stress, showing a significant increase in 442 plant biomass. At the same line, plants under stress combination showed better 443 photosynthetic performance and lower cellular oxidation than those growing under 444 salinity, with the balance between these two processes necessary for both plant growth 445 and adaptation to abiotic stress (Considine and Foyer, 2013; Woehle et al., 2017). 446 Under salinity stress, ROS accumulation (measured as $\mathrm{H}_{2} \mathrm{O}_{2}$ ) likely induced damage to 447 membranes and an increase in protein oxidation, which translated into a lower cell 448 antioxidant capacity. These oxidative stress-associated processes may have caused the 449 strong inhibition of photosynthesis and reduction of growth observed in plants subjected 450 to the salinity treatment. Interestingly, when salinity was combined with heat, ROS 451 were accumulated to a lesser extent, with the damage to membranes and proteins being 452 also lower and maintaining an antioxidant capacity of over $60 \%$, which was observed as 453 plants with better growth rates than in the salinity conditions alone. These observations 
454 indicate that ROS could be produced in a lower quantity under stress combination than 455 under salinity, and that ROS is being produced at the same level than under salinity, 456 although their detoxification may be more efficient and/or effective under stress 457 combination. Our results mainly support the latter possibility, in which the combination 458 of salinity and heat induced the reprogramming of some important stress-related 459 pathways, such as proline and ASC metabolism, facilitating their interconnectivity for a 460 more efficient cellular ROS detoxification through the activation of oxidative 461 metabolism.

462 Tomato plant responses to salinity and heat combination involved complex 463 transcriptional networks and changes in metabolic fluxes. However, the modulation of 464 the proline and ASC pathways was shown to be a strong and unique response to this 465 stress combination. Interestingly, these metabolic pathways were not found to be 466 significantly induced under salinity or heat when applied individually. Proline can 467 protect cells from damage by acting as an osmoprotectant but also as a ROS scavenger 468 (Szabados and Savouré, 2010; Narayanan and Govindarajan, 2012). Although proline 469 metabolism was induced under the combination of salinity and heat, proline levels did 470 not increase under these conditions, and instead, the derivatives 4-hydroxyproline and 471 L-glutamate-5-semialdehyde were significantly accumulated. Several studies have 472 pointed out that during stress recovery, proline is oxidized to provide the cell with a 473 large amount of energy (one molecule of proline captures 30 ATP equivalents) 474 (Verbruggen and Hermans, 2008; Zhang and Becker, 2015). Jaspers and Kangasjärvi 475 (2010) showed that when salinity levels were increased, proline was used as a source of 476 energy by plants, providing ATP and NADPH through its catalysis by the enzyme 477 PRODH. This oxidation process increased the formation of ROS, activating the 478 response signaling cascade generated by the oxidative stress (Jaspers and Kangasjärvi, 479 2010), and thus relating proline with the stress response mechanisms found in plants.

480 Our results pointed out to an interconnection between proline catalysis, ROS generation 481 (due to stress conditions and proline degradation) and an upregulation of the oxidative 482 metabolism. ASC metabolism was also up-regulated, as ASC is a necessary substrate to 483 maintain oxidative metabolism active. It can be suggested that proline accumulation 484 occurs early during the acclimation to stress combination and that its oxidation is a sign 485 of stress recovery in these plants. However, we have previously reported that proline 486 does not preferentially accumulate during the first 72 hours after tomato plants were 
487 subjected to the combination of salinity and heat stress (Rivero et al., 2014), which 488 contradicts this idea. Instead, glycine-betaine was the osmolyte that was preferentially 489 accumulated in tomato under these conditions. Thus, in this study, we propose and 490 provide evidence that proline oxidation may be interconnected with glutathione redox 491 homeostasis for efficient ROS scavenging. Recent publications have demonstrated that 492 proline catabolism to $\mathrm{P} 5 \mathrm{C}$ is induced in animal cells during cell infection (Tang and 493 Pang, 2016). These authors proposed that PRODH and PROC act together to raise P5C 494 levels and thus, govern ROS homeostasis. This mechanism is largely unknown in 495 plants, although a similar hypothesis was proposed in Arabidopsis thaliana as a 496 response to pathogen attack (Qamar et al., 2015). In a previous study by our research 497 group (Rivero et al., 2014) we have also shown that PRODH and PROC were 498 differentially up-regulated at the gene and protein levels under the combination of 499 salinity and heat, whereas under salinity or heat applied individually these enzymes 500 were down-regulated, thereby favoring proline accumulation. The significant 501 enrichment of proline metabolism found in the analysis of the transcriptomics and 502 metabolomics data and the potential role of proline intermediaries in ROS homeostasis, 503 such as P5C, provide a strong argument for the role of proline oxidation in ROS 504 signaling mechanisms under stress combination; however, we recognize that more 505 research is needed to confirm this hypothesis.

506 ASC is one of the main compounds involved in plant oxidative metabolism through the 507 Halliwell-Asada cycle, and genes and compounds found in this pathway were 508 significantly induced under salinity and heat combination. Activities of the oxidative 509 metabolism-related enzymes were determined to confirm the upregulation of the 510 oxidative metabolism at the protein level, as well as to find whether or not this pathway 511 was specifically regulated under the combination of salinity and heat, as previously 512 shown for proline and ASC metabolism. Our research group, as well as other authors, 513 have reported on the high activation of oxidative metabolism-related enzymes through a 514 specific upregulation under the combination of salinity in tomato plants (Rivero et al., 515 2014; Martinez et al., 2018; García-Martí et al., 2019). The enzymatic activities 516 assayed, together with the gene expression and the metabolites identified in our study, 517 indicate the efficient detoxification of $\mathrm{H}_{2} \mathrm{O}_{2}$ through the Halliwell-Asada cycle, and a 518 very active lipid recovery from oxidation thanks to PhGPX activity. These observations 519 could explain that under salinity and heat combination, the oxidative markers measured 
$520\left(\mathrm{H}_{2} \mathrm{O}_{2}\right.$, lipid peroxidation, protein oxidation, and antioxidant capacity) in tomato plants

521 were lower than under salinity as the sole stress.

522 Zandalinas et al. (2020) recently described that different combinations of abiotic

523 stresses applied to A. thaliana plants resulted in unique transcriptional profiles and that

524 their regulation by different $\mathrm{TF}$ families was also characteristic of each stress

525 combination. In this report, the bHLH, MYB and bZIP TFs families were significantly

526 induced under the combination of salinity and heat. Our results showed that some genes

527 belonging to the bZIP TF family were differentially and uniquely regulated under the

528 combination of salinity and heat in tomato plants (e.g., Solyclog081350). Other TFs

529 belonging to other stress-related families, such as C2H2 (e.g., Solyc02g085580,

530 Solyc03g121660, Solyc07g053570) and Trihelix (e.g., Solyc11g012720), also showed

531 this particularity under our experimental conditions. Most of these TFs families have

532 been reported to be involved in the control of plant development, cell division, different

533 physiological process, but also in abiotic responses of plants (Kaplan-Levy et al., 2014;

534 Agarwal et al., 2019). For example, Agarwal et al. (2019) reported that the bZIP family

535 was involved in the mitigation of several abiotic stresses (e.g., salinity, drought, heat or

536 oxidative stress) and the increase in plant productivity under adverse conditions. The

537 Trihelix TF family has been shown to be involved in the response to salinity and

538 pathogen-related stresses, and in the development of trichomes, stomata, and the seed

539 abscission layer (Kaplan-Levy et al., 2014). Numerous members of the C2H2-type zinc

540 finger family have been shown to play a significant role in the plant's response to

541 different abiotic stresses and in plant hormonal transduction signals (Kiełbowicz-Matuk,

542 2012). Most of the information found in the literature regarding the $\mathrm{C} 2 \mathrm{H} 2$ family has

543 been for Arabidopsis, and very little is known about other plant species, including

544 tomato. Hu et al. (2019) found that this family regulates many genes in response to

545 some abiotic stress, and especially in response to heat stress in tomato plants. Most of

546 the $\mathrm{C} 2 \mathrm{H} 2$ genes that were up-regulated under heat stress in the report by $\mathrm{Hu}$ et al.

547 (2019) were also differentially expressed in our transcriptomic analysis when heat was

548 applied as the sole stress. However, the $\mathrm{C} 2 \mathrm{H} 2$ identified in our study that was

549 specifically up-regulated under salinity + heat was not listed in the study by Hu et al.

550 (2019), again demonstrating the importance of studying stresses in combination. The

551 TFs identified as being up-regulated under the combination of salinity and heat aligned 
552 with the promoter regions of many genes studied in this report, including those

553 belonging to the proline, ASC, and oxidative metabolisms.

554 In summary, we showed that proline, ASC and oxidative metabolism are

555 interconnected, with a tight coordination to maintain not only an optimal cellular redox

556 balance, but also to trigger the proper signaling mechanisms responsible for inducing

557 the plant's acclimation to the combination of salinity and heat. In this process, proline

558 oxidation is suggested to be used as the energy source needed for triggering the stress

559 response, with subsequent ROS formation. At this point, oxidative metabolism enters

560 the stage, with the upregulation of its main enzymes to maintain ROS at basal levels.

561 One of the main limiting factors for maintaining the activity of the redox metabolic

562 pathways is ASC abundance, which suggests the presence of a connection between ASC

563 biosynthesis with oxidative metabolism and, most likely, with proline oxidation.

564 Cellular basal levels of ROS could trigger downstream signaling mechanisms through

565 the activation of particular TFs families, such as the trihelix, C2H2 and bZIP families,

566 which in turn, may regulate the expression of genes involved in the reprogramming of

567 different metabolic pathways, including those involved in proline, ASC, and redox

568 metabolism (i.e., positive feedback loops). Future validation of the role of specific TFs

569 families in the successful acclimation of plants to heat + salinity is necessary for

570 developing breeding strategies for more resilient crops against abiotic stresses.

\section{Supplementary data}

573 Figure S1. Total ion chromatogram extracted from UPLC-QTOF performed in 6 574 biological replications of tomato leaves subjected to control, salinity heat or the 575 combination of salinity + heat.

576 Figure S2. Box-and-whisker plots of the compounds belong to the Ascobate, Alderate

577 and oxidative metabolism with significant differences between salinity + heat treatment 578 respect to control.

579 Figure S3. Box-and-whisker plots of the compounds belong to the Proline metabolism

580 with significant differences between salinity + heat treatment respect to control.

581 Table S1. Raw, parsed and mapped reads of mRNA of all samples.

582 Table S2 - Sheet 1- Comparison of salinity treated tomato plants against control plants.

583 Sheet 2- Comparison of heat treated tomato plants against control plants. Sheet 3-

584 Comparison of the salinity combined with heat treatment against control plants. 
585 Table S3. Comparison of the peaks of each independent analysis with the aim of find 586 common and specific peaks among all the treatments.

587 Table S4. Identified compounds in the Control vs Salinity+Heat peaks comparison related to the enriched pathway analysis results.

589 Table S5. Activities of the oxidative metabolism-related enzymes.

590 Table S6. Differential expression output from DESeq2 (Love et al., 2014).

591 Table S7. Enrichment of KEGG pathways in upregulated genes for each treatment.

593 Data availability: The raw sequencing reads and the read mapping count matrices are 594 available in the National Center for Biotechnology Information Gene Expression 595 Omnibus database under the accession GSE152620. All data supporting the findings of 596 this study are available within the paper and within its supplementary materials 597 published online.

\section{Acknowledgements}

600 This work was supported by the Ministry of Economy and Competitiveness from Spain 601 (Grant No. PGC2018-09573-B-100) to R.M.R (Murcia, Spain), by the Ministry of 602 Science, Innovation and Universities of Spain (Grant No. FPU16/05265) to M.L-D. 603 (Murcia, Spain) and by start-up funds from the College of Agricultural and 604 Environmental Sciences and the Department of Plant Sciences (UC Davis) to B.B-U 605 (Davis, CA, United States). We sincerely acknowledge Mario G. Fon for proof-reading 606 the manuscript. We also thank the Metabolomics Core of CEBAS-CSIC for the 607 assistance with the analysis. All authors declare no commercial, industrial links or 608 affiliations.

\section{Author contribution}

611 RMR conceived, designed and supervised the experiments; ML-D, CJS and BB-U 612 performed the RNAseq analysis. VM and RMR performed the metabolomic analysis. 613 ML-D, TCM and RMR performed the biochemical analysis. ML-D and RMR 614 performed the photosynthetic measurements. ML-D, BB-U and RMR wrote the paper. 615 The authors declare that they have no competing interests. 
618 Aebi H. 1984. Catalase in Vitro. Methods in Enzymology 105, 121-126.

619 Agarwal P, Baranwal VK, Khurana P. 2019. Genome-wide Analysis of bZIP

620 Transcription Factors in wheat and Functional Characterization of a TabZIP under

621 Abiotic Stress. Scientific Reports 9, 4608.

622 Akram NA, Shafiq F, Ashraf M. 2017. Ascorbic acid-a potential oxidant scavenger

623 and its role in plant development and abiotic stress tolerance. Frontiers in Plant Science

6248.

625 Anjum SA, Xie X-Y, Wang L-C, Saleem MF, Man C, Lei W. 2019. Morphological,

626 physiological and biochemical responses of plants to drought stress. African Journal of 627 Agricultural Research 6.

628 Benjamini Y, Hochberg Y. 1995. Controlling the False Discovery Rate: A Practical 629 and Powerful Approach to Multiple Testing. Journal of the Royal Statistical Society: 630 Series B (Methodological) 57, 289-300.

631 Bolger AM, Lohse M, Usadel B. 2014. Trimmomatic: a flexible trimmer for Illumina 632 sequence data. Bioinformatics (Oxford, England) 30, 2114-2120.

633 Bradford MM. 1976. A rapid and sensitive method for the quantitation of microgram 634 quantities of protein utilizing the principle of protein-dye binding. Analytical 635 Biochemistry 72, 248-254.

636 Castelán-Muñoz N, Herrera J, Cajero-Sánchez W, Arrizubieta M, Trejo C, 637 García-Ponce B, Sánchez M de la P, Álvarez-Buylla ER, Garay-Arroyo A. 2019. 638 MADS-box genes are key components of genetic regulatory networks involved in 639 abiotic stress and plastic developmental responses in plants. Frontiers in Plant Science 64010.

641 Considine MJ, Foyer CH. 2013. Redox Regulation of Plant Development. 642 Antioxidants \& Redox Signaling 21, 1305-1326.

643 Foyer CH, Noctor G. 2011. Ascorbate and glutathione: The heart of the redox hub. 644 Plant Physiology 155, 2-18.

645 Fu J, Huang B. 2001. Involvement of antioxidants and lipid peroxidation in the 646 adaptation of two cool-season grasses to localized drought stress. Environmental and 647 Experimental Botany 45, 105-114.

648 García-Martí M, Piñero MC, García-Sanchez F, Mestre TC, López-Delacalle M, 649 Martínez V, Rivero RM. 2019. Amelioration of the Oxidative Stress Generated by 650 Simple or Combined Abiotic Stress through the $\mathrm{K}+$ and $\mathrm{Ca} 2+$ Supplementation in 
651 Tomato Plants. Antioxidants 8, 81.

652 Halliwell B, Foyer CH. 1976. Ascorbic Acid, Metal Ions and the Superoxide Radical.

653 Hoagland DR, Arnon DI. 1950. The water-culture method for growing plants without

654 soil. Circular. California Agricultural Experiment Station 347.

655 Hu X, Zhu L, Zhang Y, Xu L, Li N, Zhang X, Pan Y. 2019. Genome-wide

656 identification of $\mathrm{C} 2 \mathrm{H} 2$ zinc-finger genes and their expression patterns under heat stress

657 in tomato (Solanum lycopersicum L.) (G Sun, Ed.). PeerJ 7, e7929.

658 Jaspers P, Kangasjärvi J. 2010. Reactive oxygen species in abiotic stress signaling.

659 Physiologia Plantarum 138, 405-413.

660 Kaplan-Levy RN, Quon T, O'Brien M, Sappl PG, Smyth DR. 2014. Functional

661 domains of the PETAL LOSS protein, a trihelix transcription factor that represses

662 regional growth in Arabidopsis thaliana. The Plant Journal 79, 477-491.

663 Kiełbowicz-Matuk A. 2012. Involvement of plant C2H2-type zinc finger transcription 664 factors in stress responses. Plant Science 185-186, 78-85.

665 Koleva II, van Beek TA, Linssen JPH, Groot A de, Evstatieva LN. 2002. Screening 666 of Plant Extracts for Antioxidant Activity: a Comparative Study on Three Testing 667 Methods. Phytochemical Analysis 13, 8-17.

668 Kollist H, Zandalinas SI, Sengupta S, Nuhkat M, Kangasjärvi J, Mittler R. 2019.

669 Rapid Responses to Abiotic Stress: Priming the Landscape for the Signal Transduction 670 Network. Trends in Plant Science 24, 25-37.

671 Langmead B, Salzberg SL. 2012. Fast gapped-read alignment with Bowtie 2. Nature 672 methods $\mathbf{9}, 357-359$.

673 Lopez-Delacalle M, Camejo D, ... MG-M-F in P, 2019 U. 2020. Using tomato 674 recombinant lines to improve plant tolerance to stress combination through a more 675 efficient nitrogen metabolism. Frontiers.

676 Love MI, Huber W, Anders S. 2014. Moderated estimation of fold change and 677 dispersion for RNA-seq data with DESeq2. Genome Biology 15, 550.

678 MacNevin WM, Urone PF. 1953. Separation of Hydrogen Peroxide from Organic 679 Hydroperoxides. Analytical Chemistry 25, 1760-1761.

680 Martinez V, Mestre TC, Rubio F, Girones-Vilaplana A, Moreno DA, Mittler R, 681 Rivero RM. 2016. Accumulation of flavonols over hydroxycinnamic acids favors 682 oxidative damage protection under abiotic stress. Frontiers in Plant Science 7.

683 Martinez V, Nieves-Cordones M, Lopez-Delacalle M, Rodenas R, Mestre TC, 
684 Garcia-Sanchez F, Rubio F, Nortes PA, Mittler R, Rivero RM. 2018. Tolerance to 685 stress combination in tomato plants: New insights in the protective role of melatonin. 686 Molecules 23, 1-20.

687 McCord JM FI. 1969. The utility of superoxide Dismutase in studyng free Radicals 688 Reactions. The Journal of biological chemistry 244, 6056 - 63.

689 McLeay RC, Bailey TL. 2010. Motif Enrichment Analysis: a unified framework and 690 an evaluation on ChIP data. BMC Bioinformatics 11, 165.

691 Meyer AJ. 2008. The integration of glutathione homeostasis and redox signaling. 692 Journal of Plant Physiology 165, 1390-1403.

693 Miller G, Suzuki N, Ciftci-Yilmaz S, Mittler R. 2010. Reactive oxygen species 694 homeostasis and signalling during drought and salinity stresses. Plant, Cell and 695 Environment 33, 453-467.

696 Mittler R. 2006. Abiotic stress, the field environment and stress combination. Trends in 697 Plant Science.

698 Miyake C, Asada K. 1992. Thylakoid-Bound Ascorbate Peroxidase in Spinach 699 Chloroplasts and Photoreduction of Its Primary Oxidation Product 700 Monodehydroascorbate Radicals in Thylakoids. Plant and Cell Physiology 33, 541-553.

701 Moriya Y, Itoh M, Okuda S, Yoshizawa AC, Kanehisa M. 2007. KAAS: an 702 automatic genome annotation and pathway reconstruction server. Nucleic Acids 703 Research 35, W182-W185.

704 Narayanan N, Govindarajan SK. 2012. Natarajan. N., and G. Suresh Kumar. (2012).

705 "Effect of non linear sorption on multi-species solute transport in a coupled fracture 706 matrix system". International Journal of Research in Chemistry and Environment 707 (ISSN: 2248-9649), Vol. 2(2), pp. 96-101. International Journal of Research in 708 Chemistry and Environment 2, 96-101.

709 Nieves-Cordones M, López-Delacalle M, Ródenas R, Martínez V, Rubio F, Rivero

710 RM. 2019. Critical responses to nutrient deprivation: A comprehensive review on the 711 role of ROS and RNS. Environmental and Experimental Botany 161, 74-85.

712 Noctor G, Foyer CH. 1998. ASCORBATE AND GLUTATHIONE: Keeping Active 713 Oxygen Under Control. Annual Review of Plant Physiology and Plant Molecular 714 Biology 49, 249-279.

715 Qamar A, Mysore KS, Senthil-Kumar M. 2015. Role of proline and pyrroline-5716 carboxylate metabolism in plant defense against invading pathogens. Frontiers in plant 
science 6, 503 .

Reznick AZ, Packer L. 1994. [38] Oxidative damage to proteins: Spectrophotometric method for carbonyl assay. Methods in Enzymology. Academic Press, 357-363.

\section{Rivera A, Bravo C, Buob G. 2017. Climate Change and Land Ice.}

\section{Rivero RM, Mestre TC, Mittler R, Rubio F, Garcia-Sanchez F, Martinez V. 2014.}

The combined effect of salinity and heat reveals a specific physiological, biochemical and molecular response in tomato plants. Plant, Cell and Environment 37, 1059-1073.

Rivero RM, Ruiz JM, Romero L. 2004a. Oxidative metabolism in tomato plants subjected to heat stress. Journal of Horticultural Science and Biotechnology 79, 560564.

Rivero RM, Ruiz JM, Romero LM. 2004b. Importance of N source on heat stress tolerance due to the accumulation of proline and quaternary ammonium compounds in tomato plants. Plant Biology 6, 702-707.

Sayed HESA El. 2013. Exogenous application of ascorbic acid for improve germination, growth, water relations, organic and inorganic components in tomato (Lycopersicon esculentum Mill.). New York Science Journal 6, 123-139.

Schmidt R, Schippers JHM, Welker A, Mieulet D, Guiderdoni E, Mueller-Roeber B. 2012. Transcription factor oshsfclb regulates salt tolerance and development in oryza sativa ssp. japonica. AoB PLANTS 12.

Sehgal A, Sita K, Bhandari K, Kumar S, Kumar J, Vara Prasad P V, Siddique KHM, Nayyar H. 2019. Influence of drought and heat stress, applied independently or in combination during seed development, on qualitative and quantitative aspects of seeds of lentil (Lens culinaris Medikus) genotypes, differing in drought sensitivity. Plant Cell and Environment 42, 198-211.

Shalata A, Neumann PM. 2001. Exogenous ascorbic acid (vitamin C) increases resistance to salt stress and reduces lipid peroxidation. Journal of Experimental Botany 52, 2207-2211.

Suzuki N, Koussevitzky S, Mittler R, Miller G. 2012. ROS and redox signalling in the response of plants to abiotic stress. Plant, Cell and Environment 35, 259-270.

Szabados L, Savouré A. 2010. Proline: a multifunctional amino acid. Trends in Plant Science 15, 89-97.

Tang H, Pang S. 2016. Proline Catabolism Modulates Innate Immunity in Caenorhabditis elegans. Cell Reports 17, 2837-2844. 
750 Torres CA, Andrews PK, Davies NM. 2006. Physiological and biochemical responses

751 of fruit exocarp of tomato (Lycopersicon esculentum Mill.) mutants to natural photo-

752 oxidative conditions. Journal of Experimental Botany.1933-1947.

753 Verbruggen N, Hermans C. 2008. Proline accumulation in plants: a review. Amino 754 Acids 35, 753-759.

755 Woehle C, Dagan T, Landan G, Vardi A, Rosenwasser S. 2017. Expansion of the 756 redox-sensitive proteome coincides with the plastid endosymbiosis. Nature Plants $\mathbf{3}$, $757 \quad 17066$.

758 Zandalinas SI, Fritschi FB, Mittler R, Lawson T. 2020. Signal transduction networks 759 during stress combination. Journal of Experimental Botany 71, 1734-1741.

760 Zhang L, Becker D. 2015. Connecting proline metabolism and signaling pathways in 761 plant senescence. Frontiers in Plant Science 6, 552.

762 Zushi K, Ono M, Matsuzoe N. 2014. Light intensity modulates antioxidant systems in 763 salt-stressed tomato (Solanum lycopersicum L. cv. Micro-Tom) fruits. Scientia 764 Horticulturae 165, 384-391.

\section{Figure Legends}

Figure 1. (A) Pictures of tomato plants at the end of the control or stress treatments. (B) Whole plant fresh weight (FW) of tomato plants grown under control, heat, salinity or the combination of salinity and heat. (C-F) Photosynthetic parameters measured in the third and four fully mature expanded leaves of tomato plants grown under control or stress conditions measured at the beginning (0 days), during (7 days) or at the end (14 days) of the treatments. Values represent means \pm SE $(n=9)$. Bars with different letters within each panel are significantly different at $\mathrm{p}<0.05$ according to Tukey's test.

Figure 2. RNAseq analysis performed in tomato leaves after 14 days of growing under control or stress (salinity, heat or the combination of salinity and heat) conditions. (A) Principal component analysis (PCA) of the normalized reads obtained for each treatment. (B) Euler diagram representing up- and down-regulated genes (adjusted $\mathrm{P}<0.05$ ) of tomato plants grown under control, simple (salinity or heat) or combined (salinity + heat) stress. (C) Pathway enrichment analysis performed within the up-regulated genes under the different stress conditions applied. More 
details on these analyses can be found in the Materials and Method section.

Figure 3. Metabolomic analysis performed in tomato leaves after 14 days of growing under control, simple (salinity or heat) or combined (salinity + heat) stress conditions. (A) Principal component analysis (PCA) of the normalized molecular features found under each treatment applied $(n=6)$. (B) Euler diagram of the common and uniquely molecular features with a differential and significant accumulation in each treatment $\left(P_{a d j}<0.05\right)$. (C) Bubble diagram representing the up- and down-regulated molecular features found among the 568 molecular features uniquely and significantly changing under the combination of salinity + heat. Each bubble (i.e. molecular feature) is positioned in the chromatogram by its mass-to-charge (y-axis) and retention time (x-axis) and the size and darkness of one bubble represented the $\log _{2}$ and p-value, respectively of this molecular feature. The raw data of Figure $3 \mathrm{C}$ can be found in the supplemental material (Supplementary Table S2 and S3). (D) KEGG pathway enrichment analysis performed with the significantly up-regulated molecular features identified under the combination of salinity + heat. More details on these analyses can be found in the Materials and Methods section.

Figure 4. Schematic diagram of the metabolic interconnection between ascorbate, proline and oxidative metabolism in tomato plants. $\log _{2}$ (fold change) of the metabolite concentration $(\circ)$, gene expression $(\square \square)$ or enzymatic activity $(\diamond)$ obtained in tomato plants grown under the combination of salinity + heat after RNAseq, metabolomics or biochemical analyses were represented.

Figure 5. Oxidative metabolism-related markers measured in tomato leaves grown for 14 days under control, simple (salinity theat) or combined (salinity+heat) stress. Values represent means \pm SE $(n=9)$. Bars with different letters within each panel are significantly different at $\mathrm{p}<0.05$ according to Tukey's test.

Figure 6. Cis-element enrichment results for up-regulated genes from each stress treatment. (A) Enrichment p-values for binding motifs corresponding to 46 TFs in each of the stress treatments. TFs are grouped and color coded by family, and a consensus diagram for the binding motif and gene accession is given for each. (B) $\log _{2}$ (fold change) of expression of three selected TFs in each stress treatment. (C) Counts of TF families overrepresented in genes up-regulated in the salinity + heat treatment from the ascorbate metabolism, oxidative metabolism, and proline 
bioRxiv preprint doi: https://doi.org/10.1101/2020.06.30.179770; this version posted July 2, 2020. The copyright holder for this preprint (which was not certified by peer review) is the author/funder. All rights reserved. No reuse allowed without permission.

816 metabolism families. Accessions and common abbreviations are given for each

817 gene. Numbers in boxes refer to the count of TFs in that family with a match to that gene based on Analysis of Motif Enrichment results.

819 
A

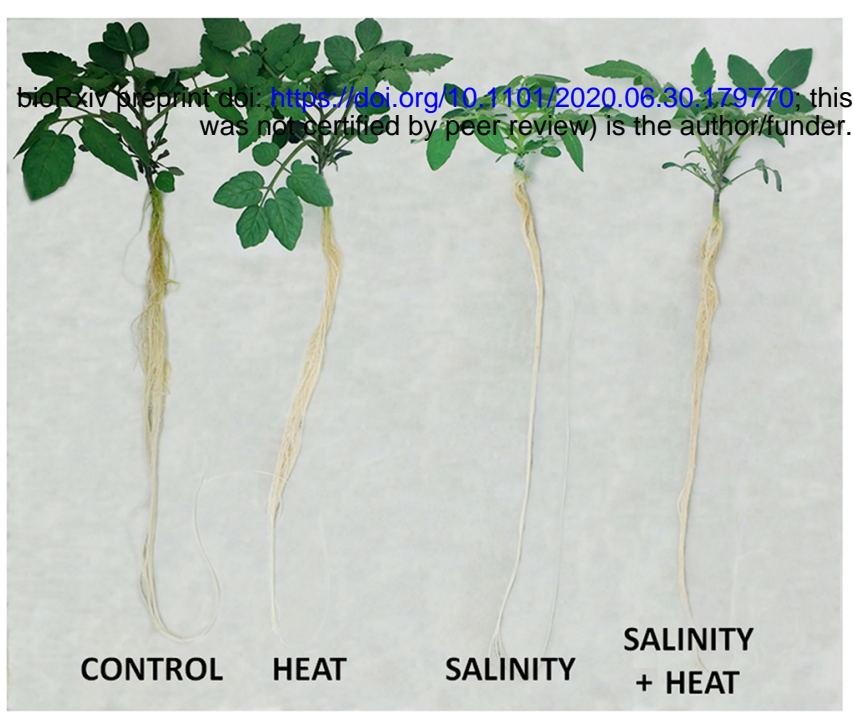

C $\mathrm{CO}_{2}$ Assimilation rate

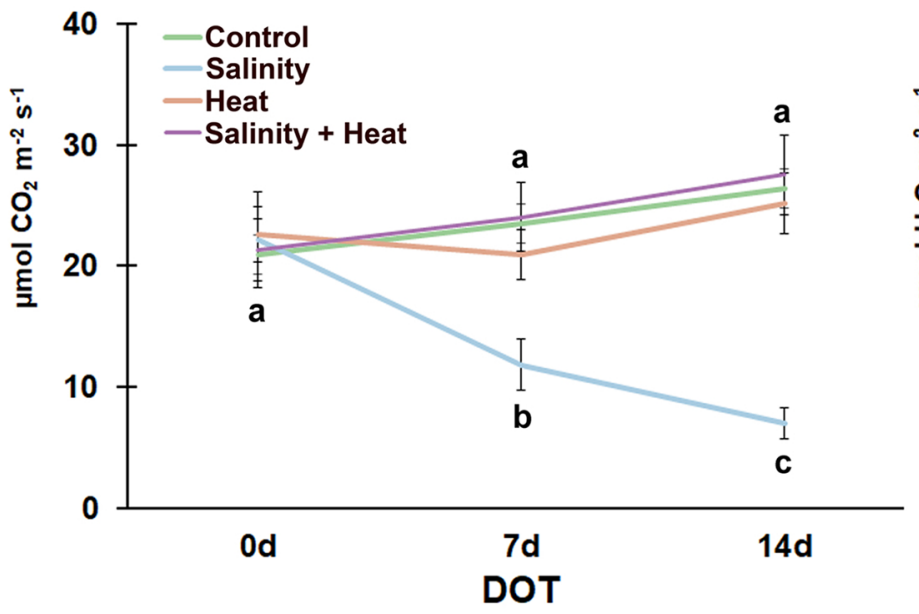

E

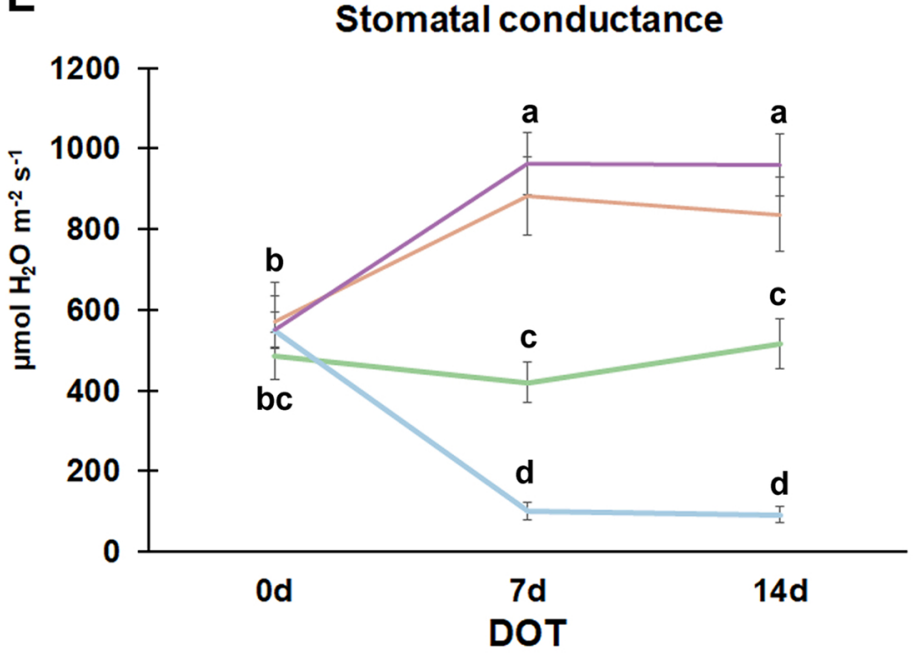

D

$\mathbf{F}$
B

Eresh weigh

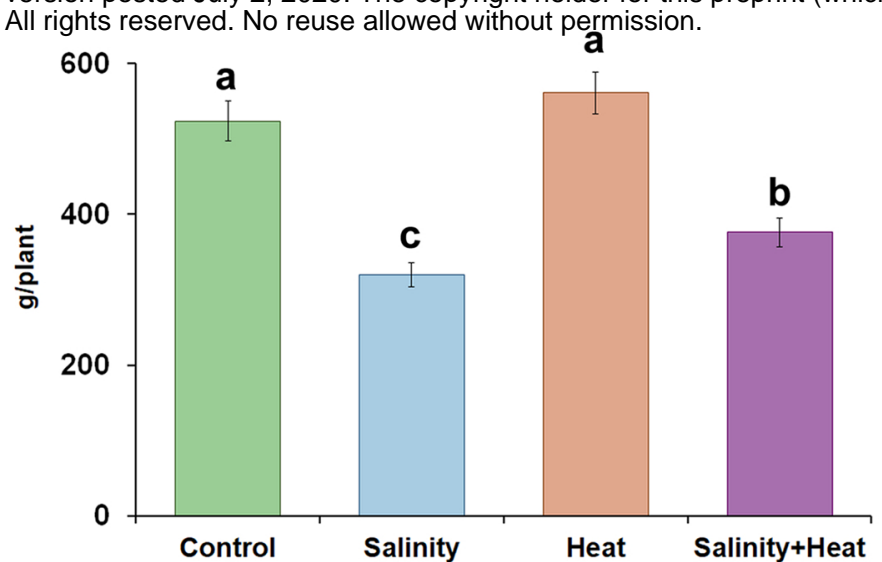
Transpiration rate

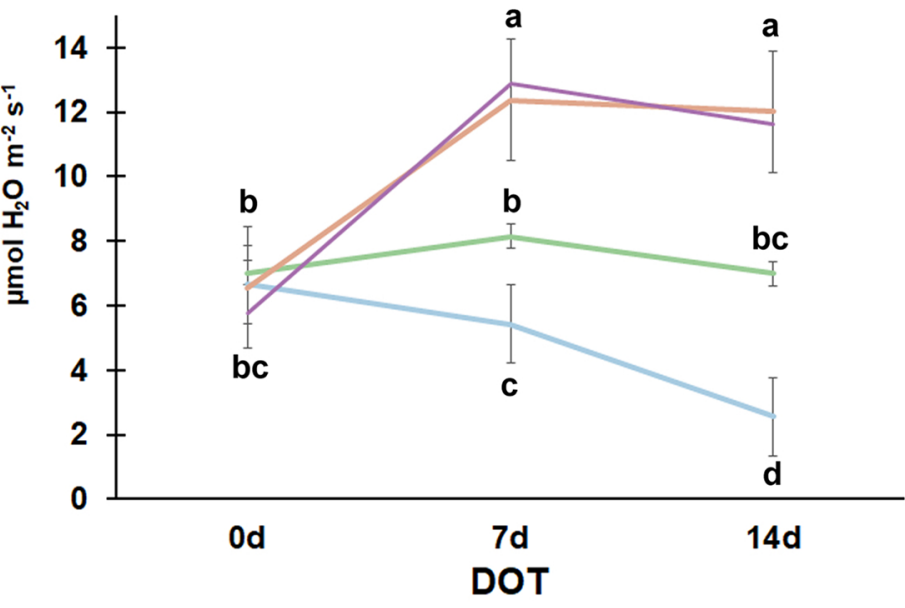

WUE

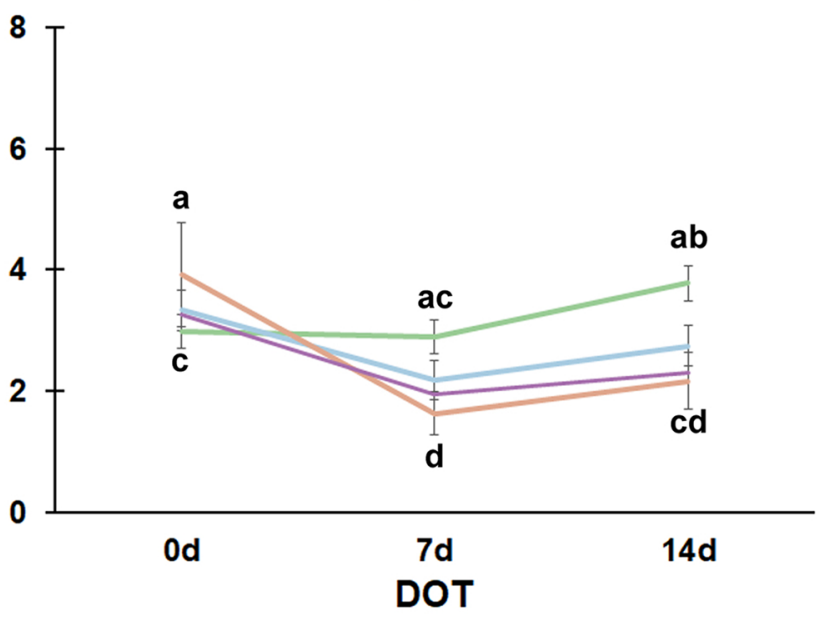

Figure 1. (A) Pictures of tomato plants at the end of the control or stress treatments. (B) Whole plant fresh weight (FW) of tomato plants grown under control, heat, salinity or the combination of salinity and heat. (C-F) Photosynthetic parameters measured in the third and four fully mature expanded leaves of tomato plants grown under control or stress conditions measured at the beginning ( 0 days), during ( 7 days) or at the end (14 days) of the treatments. Values represent means \pm SE ( $n$ $=9$ ). Bars with different letters within each panel are significantly different at $p<0.05$ according to Tukey's test. 


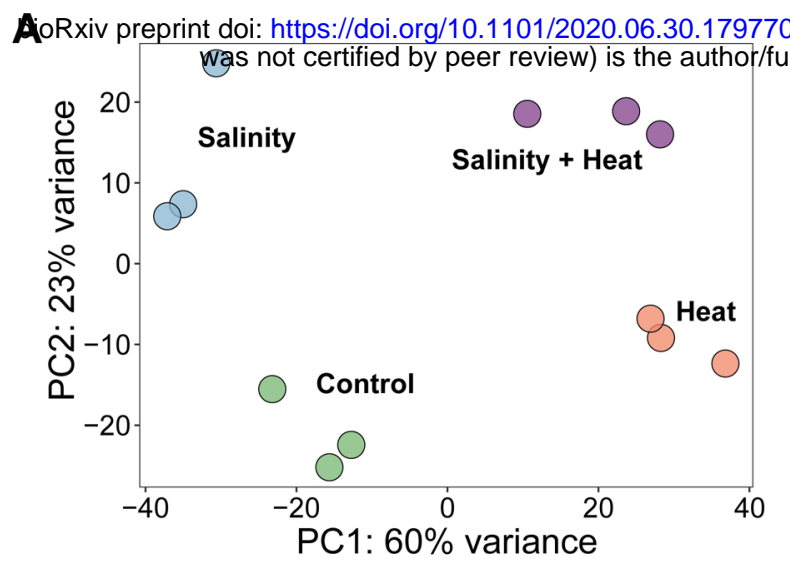

B

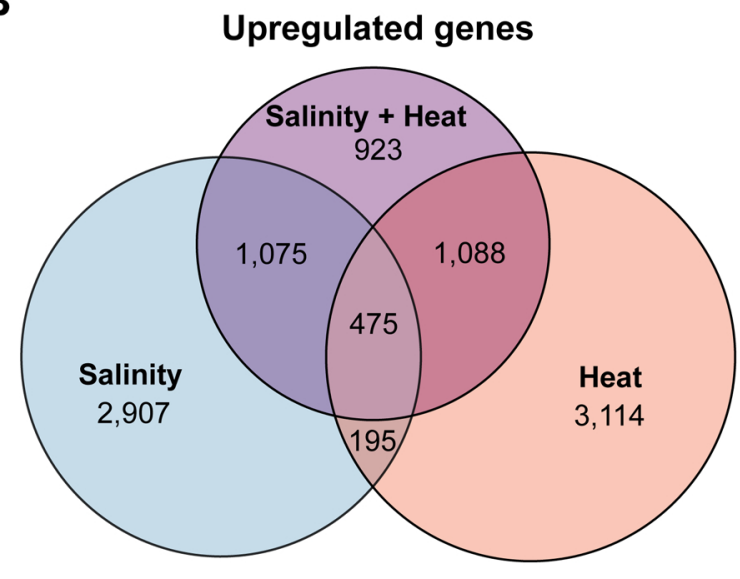

\section{Downregulated genes}

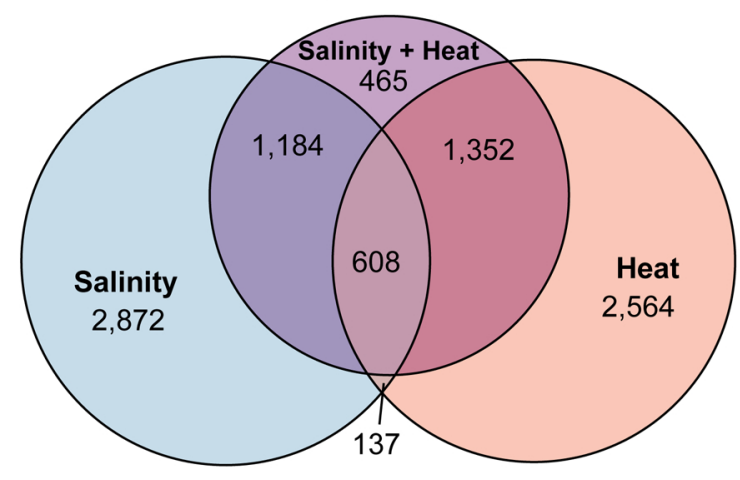

version posted July 2, 2020. The copyright holder for this preprint (which version posted July 2,2020 . The copyright holder for this
All rights reserved. No reuse allowed without permissio

$$
\begin{aligned}
& \text { Ribosome (sly03010) } \\
& \text { Oxidative phosphorylation (sly00190) - } 50 \\
& \text { Ubiquitin mediated proteolysis (sly04120) - } 47 \\
& \text { Glutathione metabolism (sly00480) - } 35 \\
& \text { Phagosome (sly04145) - } 29 \\
& \text { Protein processing in ER (sly04141) - } \begin{array}{lll}
54 & 60 & 41
\end{array} \\
& \text { GPI-anchor biosynthesis (sly00563) - } 13 \\
& \text { Autophagy - other (sly04136) - } 15 \\
& \text { Plant hormone signal transduction (sly04075) - } 62 \\
& \text { Spliceosome (sly03040) - } 43 \\
& \text { mRNA surveillance pathway (sly03015) - } \\
& \text { RNA transport (sly03013) - } \\
& \text { Ribosome biogenesis in eukaryotes (sly03008) - } \\
& \text { Starch and sucrose metabolism (sly00500) - } \\
& \text { Endocytosis (sly04144) - } \\
& \text { RNA degradation (sly03018) - } \\
& \text { Circadian rhythm (sly04712) - } \\
& \text { Aminoacyl-tRNA biosynthesis (sly00970) - } 18 \\
& \text { Other types of O-glycan biosynthesis (sly00514) - } 8 \\
& \text { Inositol phosphate metabolism (sly00562)- } 22 \\
& \text { Pyruvate metabolism (sly00620) - } 26 \\
& \text { Homologous recombination (sly03440)- } 20 \\
& \text { RNA polymerase (sly03020)- } 15 \\
& \text { Base excision repair (sly03410) - } 15 \\
& \text { Non-homologous end-joining (sly03450)- } 6 \\
& \text { Ascorbate and aldarate metabolism (sly00053) - } 16 \\
& \text { Arginine and proline metabolism (sly00330) - } \\
& \begin{array}{llll}
\log _{10}\left(1 / \mathrm{P}_{\text {adj }}\right) & \\
2.5 & 5 & 7.5 & 10
\end{array}
\end{aligned}
$$

Figure 2. RNAseq analysis performed in tomato leaves after 14 days of growing under control or stress (salinity, heat or the combination of salinity and heat) conditions. (A) Principal component analysis (PCA) of the normalized reads obtained for each treatment. (B) Euler diagram representing up- and down-regulated genes (adjusted $\mathrm{P}<0.05$ ) of tomato plants grown under control, simple (salinity or heat) or combined (salinity + heat) stress. (C) Pathway enrichment analysis performed within the up-regulated genes under the different stress conditions applied. More details on these analyses can be found in the Materials and Method section. 
A

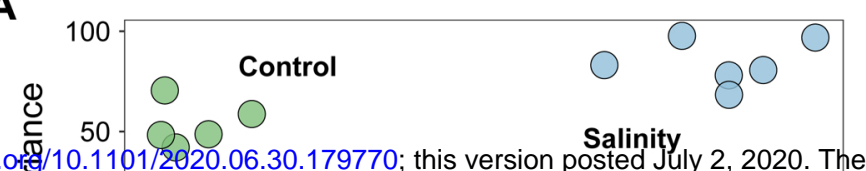

bioRxiv preprint doi: https://doi.o畄/10.1101/2020.06.30.179770; this version posted Sality 2, 2020. The copyright holder for this preprint (which was not certified bey peer review) is the author/funder. All rights reserved. No reuse allowed without permission.

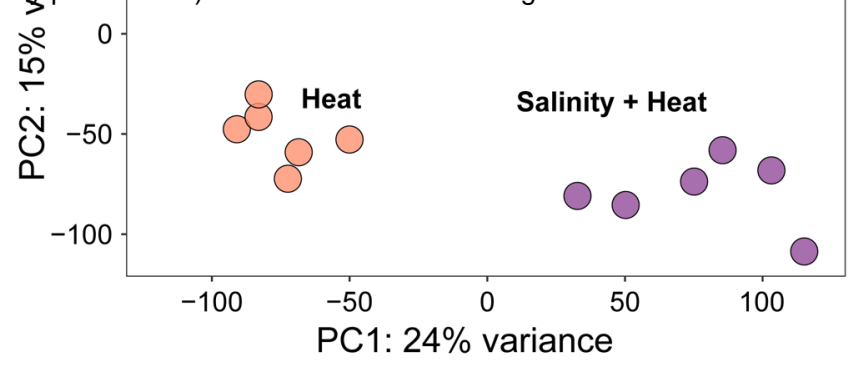

B

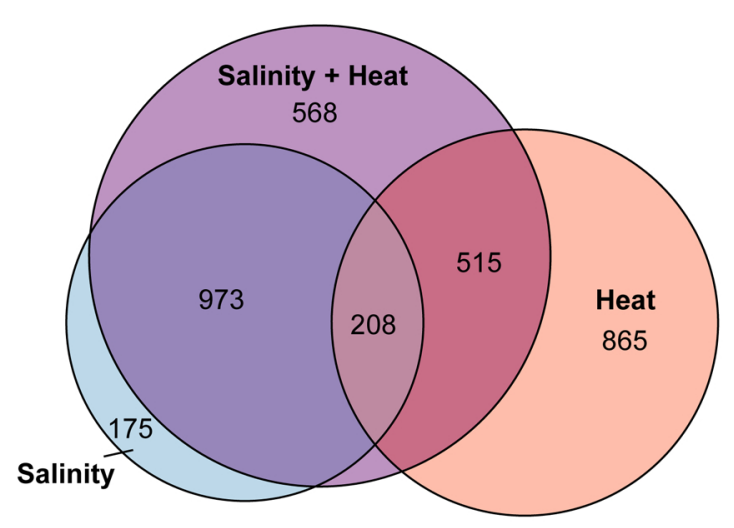

C
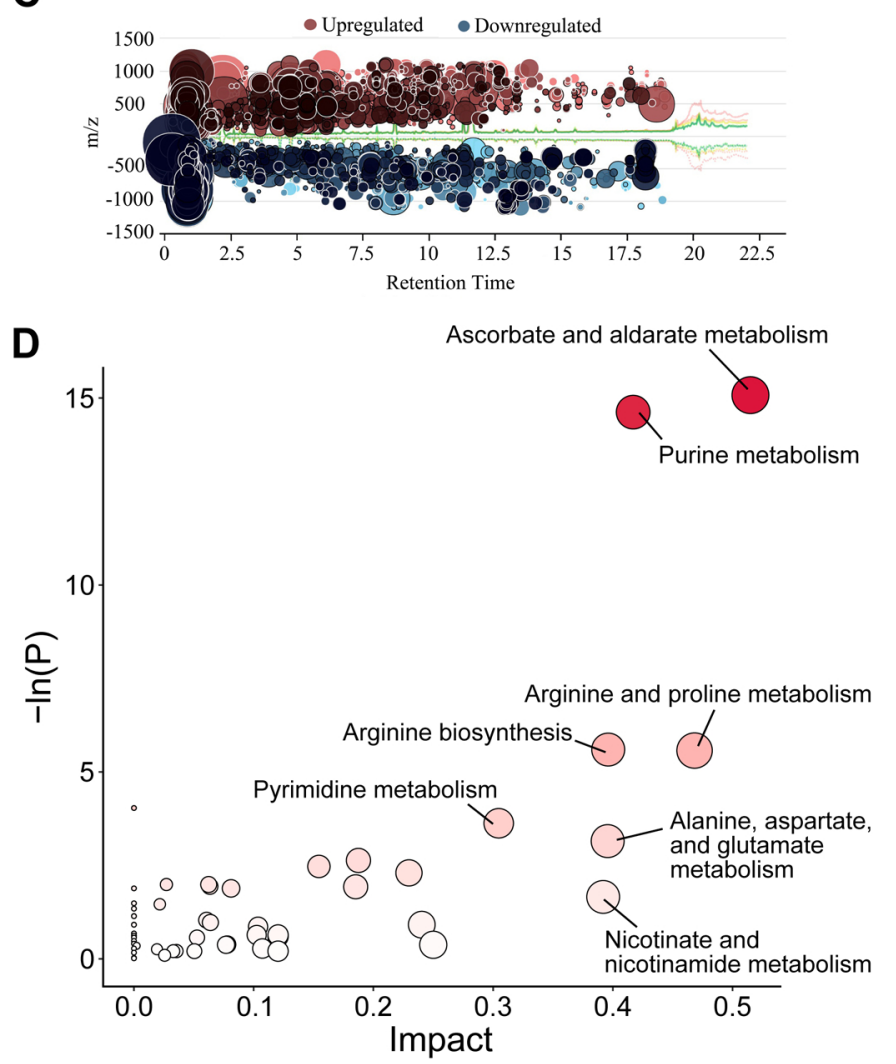

Figure 3. Metabolomic analysis performed in tomato leaves after 14 days of growing under control, simple (salinity or heat) or combined (salinity + heat) stress conditions. (A) Principal component analysis (PCA) of the normalized molecular features found under each treatment applied $(n=6)$. (B) Euler diagram of the common and uniquely molecular features with a differential and significant accumulation in each treatment (Padj < 0.05). (C) Bubble diagram representing the up- and down-regulated molecular features found among the 568 molecular features uniquely and significantly changing under the combination of salinity + heat. Each bubble (i.e. molecular feature) is positioned in the chromatogram by its mass-to-charge ( $y$-axis) and retention time (x-axis) and the size and darkness of one bubble represented the log2 and $p$-value, respectively of this molecular feature. The raw data of Figure 3C can be found in the supplemental material (Supplementary Table S2 and S3). (D) KEGG pathway enrichment analysis performed with the significantly up-regulated molecular features identified under the combination of salinity + heat. More details on these analyses can be found in the Materials and Methods section. 
bioRxiv preprint doi: https://doi.org/10.1101/2020.06.30.179770; this version posted July 2, 2020. The copyright holder for this preprint (which was not certified by peer review) is the author/funder. All rights reserved. No reuse allowed without permission.

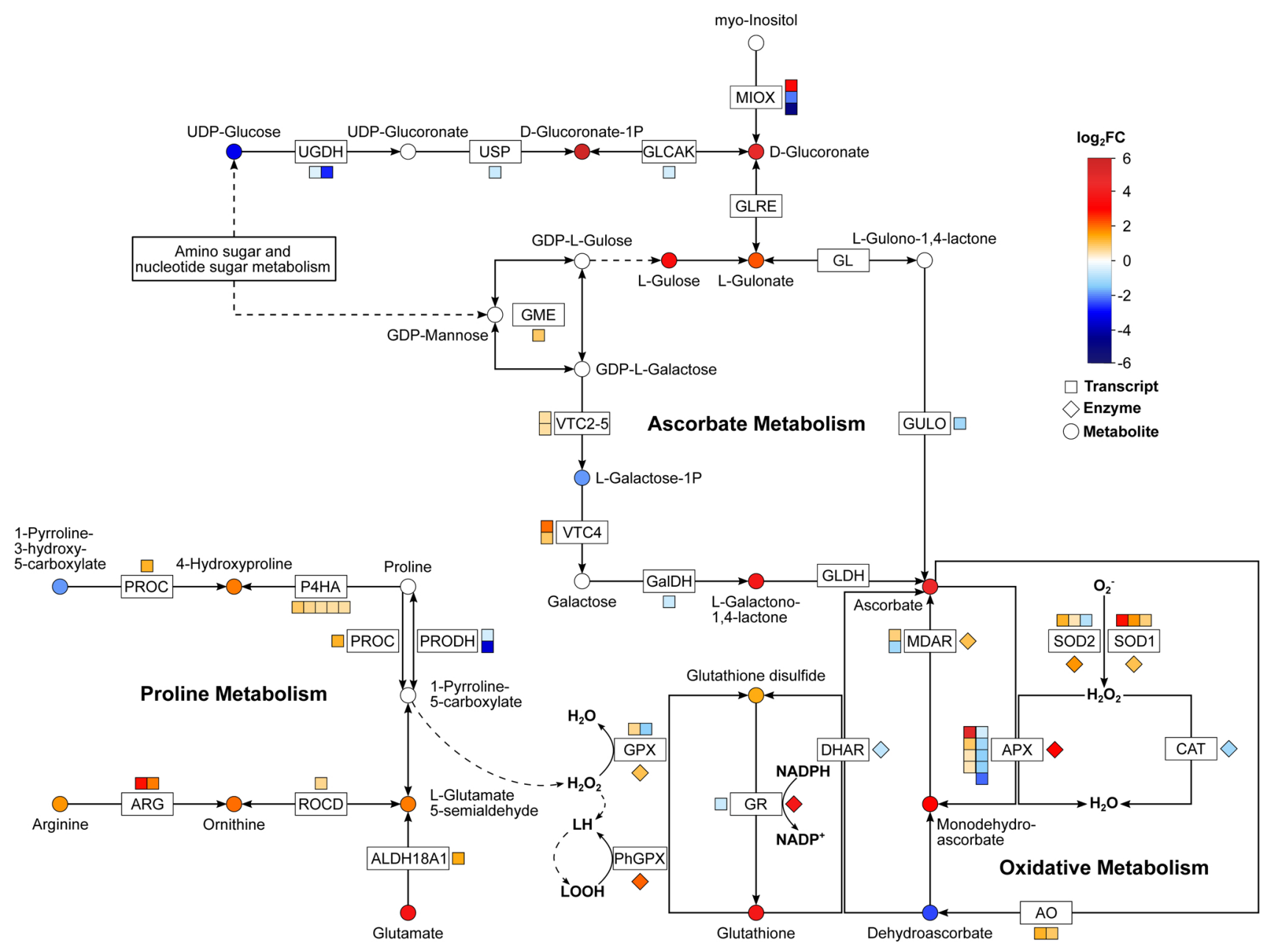

Figure 4. Schematic diagram of the metabolic interconnection between ascorbate, proline and oxidative metabolism in tomato plants. Log2(fold change) of the metabolite concentration (०), gene expression (??) or enzymatic activity $(\diamond)$ obtained in tomato plants grown under the combination of salinity + heat after RNAseq, metabolomics or biochemical analyses were represented. 


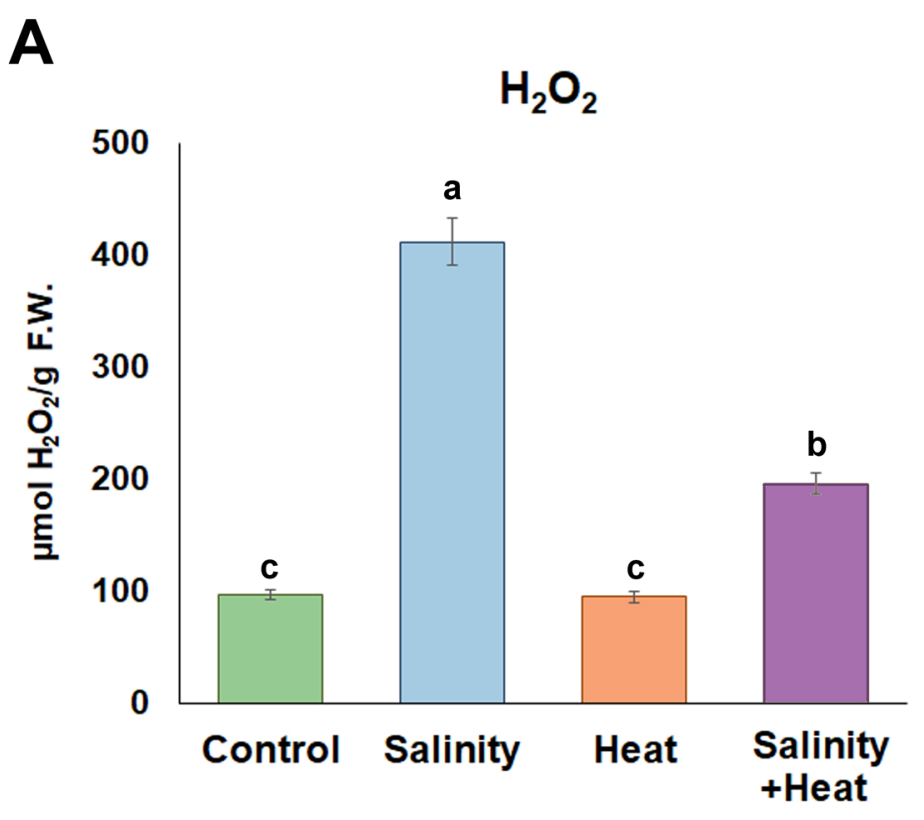

B
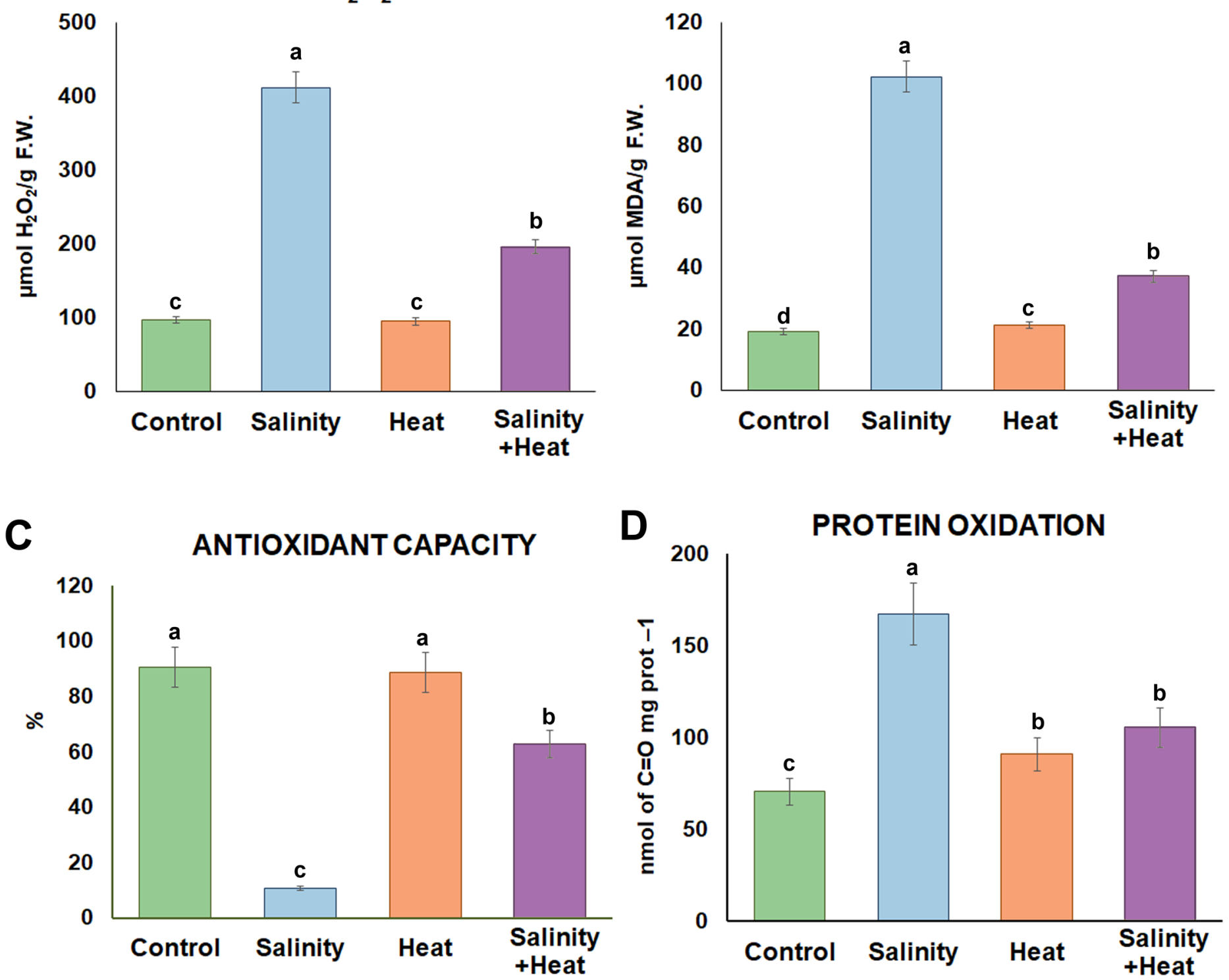

Figure 5. Oxidative metabolism-related markers measured in tomato leaves grown for 14 days under control, simple (salinity theat) or combined (salinity+heat) stress. Values represent means $\pm S E(n=9)$. Bars with different letters within each panel are significantly different at $p<0.05$ according to Tukey's test. 


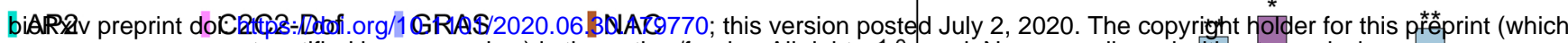

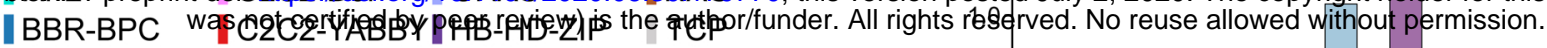

$\begin{array}{llll}\text { bHLH } & \mathrm{C} 2 \mathrm{H} 2 & \text { MYB } & \text { Trihelix } \\ \text { IbZIP } & \text { ICPP } & \text { MYB-related } & \text { |zf-HD }\end{array}$
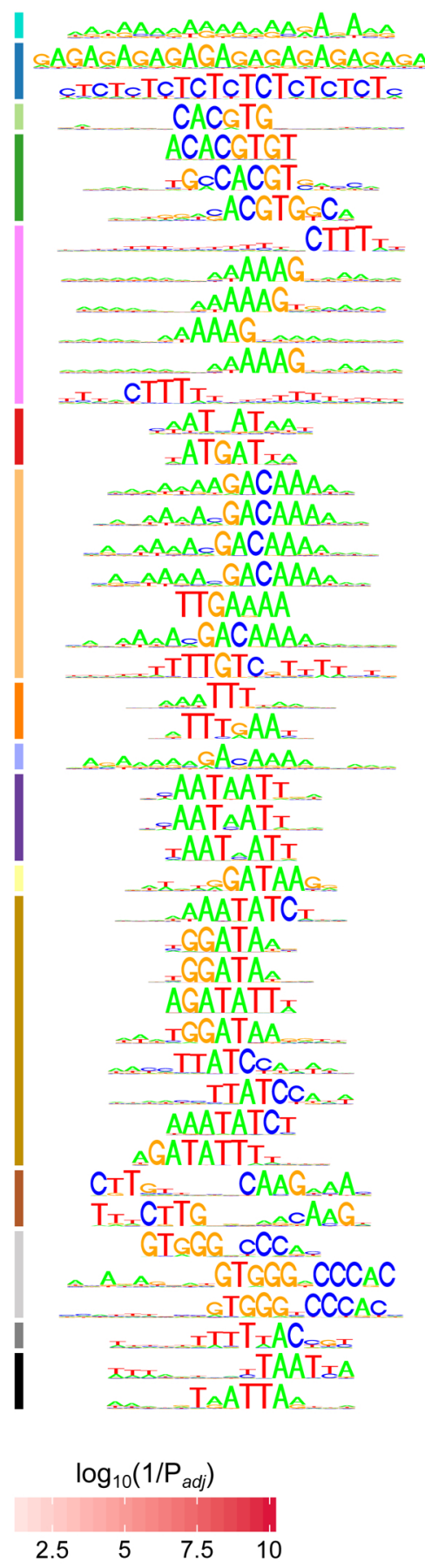

Solyc11g008560 Solyc04g008380 Solyc04g081170 Solyc05g006650 Solyc01g108080 Solyc04g078840 Solyc10g081350 Solyc02g090220 Solyc03g112930 Solyc03g115940 Solyc06g062520 Solyc06g071480 Solyc06g076030 Solyc01g091010 Solyc05g012050 Solyc01g005060 Solyc02g085580 Solyc03g121660 Solyc04g008500Solyc04g015570 Solyc07g053570 Solyc10g084180 Solyc09g082500 Solyc12g007180 Solyc11g011260 Solyc02g086930 Solyc05g007180 Solyc05g051460 Solyc04g008870 Solyc02g036370 Solyc03g113620 Solyc04g005100 Solyc06g036300 Solyc06g071230 Solyc07g026680 Solyc08g078340Solyc10g005080 Solyc10g084370 Solyc07g063420 Solyc11g008000 Solyc04g009180 Solyc06g070900 Solyc08g080150 Solyc11g012720 Solyc02g067310 Solyc02g085160

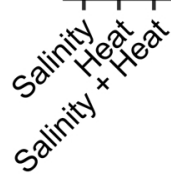

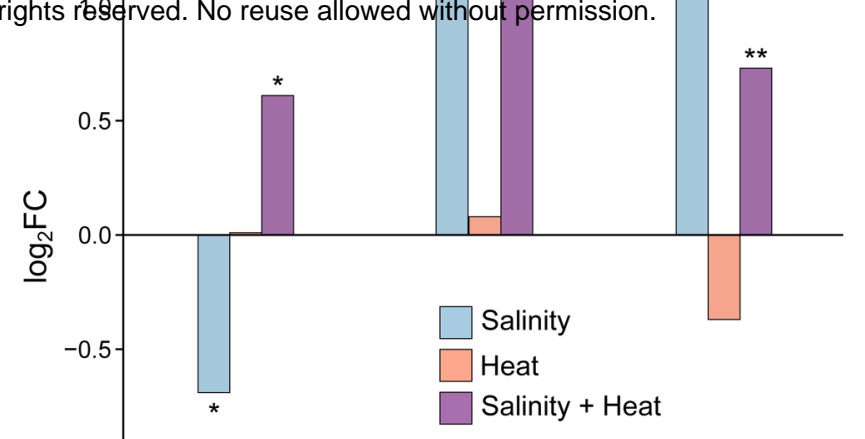

C

Ascorbate Metabolism AO Solyc01g008830 Solyc01g056310 Solyc06g005150 Solyc06g005160 Solyc06g060260 Solyc09g007270

GME | Solyc01g097340 MDAR | Solyc02g086710 MIOX | Solyc06g062430 VTC2-5 Solyc02g091510 Solyc06g073320 VTC4 $\mid$\begin{tabular}{l|l} 
Solyc03g044890 \\
Solyc11g012410
\end{tabular} GPX | Solyc12g056240
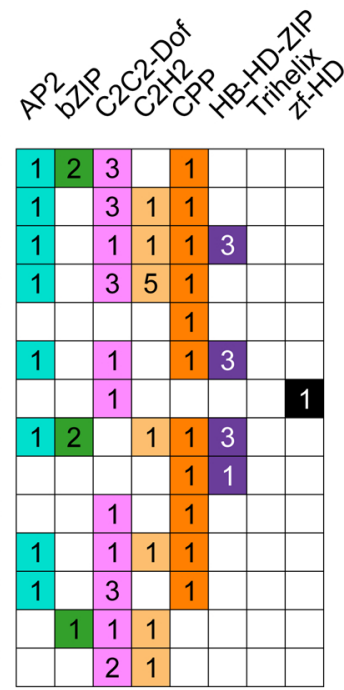

Oxidative Metabolism

\begin{tabular}{|c|c|c|c|c|c|c|c|}
\hline \multirow{3}{*}{ SOD1 } & Solyc01g067740 & & & 1 & 1 & 1 & \\
\hline & Solyc03g062890 & & 2 & & & & \\
\hline & Solyc11g066390 & & 3 & 1 & & 3 & \\
\hline \multirow{2}{*}{ SOD2 } & Solyc02g021140 & 1 & 1 & 1 & & 3 & \\
\hline & Solyc03g095180 & 1 & 3 & & 1 & & \\
\hline
\end{tabular}

\section{Proline Metabolism}

ALDH18A1 | Solyc06g019170

ARG $\mid$\begin{tabular}{l|l} 
Solyc01g091160 \\
Solyc01g091170
\end{tabular} Solyc01g080530 Solyc02g092710

P4HA Solyc04g081930 Solyc06g054490 Solyc11g005200 PROC | Solyc02g068640

Figure 6. Cis-element enrichment results for up-regulated genes from each stress treatment. (A) Enrichment $p$-values for binding motifs corresponding to 46 TFs in each of the stress treatments. TFs are grouped and color coded by family, and a consensus diagram for the binding motif and gene accession is given for each. (B) $\log _{2}$ (fold change) of expression of three selected TFs in each stress treatment. (C) Counts of TF families overrepresented in genes up-regulated in the salinity + heat treatment from the ascorbate metabolism, oxidative metabolism, and proline metabolism families. Accessions and common abbreviations are given for each gene. Numbers in boxes refer to the count of TFs in that family with a match to that gene based on Analysis of Motif Enrichment results. 